\title{
NP1 Regulates Neuronal Activity-Dependent Accumulation of BAX in Mitochondria and Mitochondrial Dynamics
}

\author{
Kevin B. Clayton, ${ }^{1,3 \star}$ Petar Podlesniy, ${ }^{1,3 *}$ Joana Figueiro-Silva, ${ }^{1,3}$ Guillermo López-Doménech, ${ }^{2,3}$ Lluis Benitez, ${ }^{1,3}$ \\ Marta Enguita, ${ }^{1}$ Maria A. Abad, ${ }^{3}$ Eduardo Soriano, ${ }^{2,3}$ and Ramon Trullas ${ }^{1,3}$ \\ ${ }^{1}$ Neurobiology Unit, Biomedical Research Institute of Barcelona, Consejo Superior de Investigaciones Científicas, Institute of Biomedical Research August \\ Pi i Sunyer, 08036 Barcelona, Spain, ${ }^{2}$ Institute for Research in Biomedicine, Barcelona Science Park, 08028 Barcelona, Spain, and ${ }^{3}$ Biomedical Research \\ Center Network on Neurodegenerative Diseases, 28049 Madrid, Spain
}

In cultured cerebellar granule neurons, low neuronal activity triggers the intrinsic program of apoptosis, which requires protein synthesis-dependent BAX translocation to mitochondria, a process that may underlie neuronal damage in neurodegeneration. However, the mechanisms that link neuronal activity with the induction of the mitochondrial program of apoptosis remain unclear. Neuronal pentraxin 1 (NP1) is a pro-apoptotic protein induced by low neuronal activity that is increased in damaged neurites in Alzheimer's disease-affected brains. Here we report that NP1 facilitates the accumulation of BAX in mitochondria and regulates mitochondrial dynamics during apoptosis in rat and mouse cerebellar granule neurons in culture. Reduction of neuronal activity increases NP1 protein levels in mitochondria and contributes to mitochondrial fragmentation in a Bax-dependent manner. In addition, NP1 is involved in mitochondrial transport in healthy neurons. These results show that NP1 is targeted to mitochondria acting upstream of BAX and uncover a novel function for NP1 in the regulation of mitochondrial dynamics and trafficking during apoptotic neurodegeneration.

\section{Introduction}

In mature neurons, low neuronal activity triggers the intrinsic program of apoptotic cell death (D’Mello et al., 1993). One of the proteins that links neuronal activity with the apoptotic program in differentiated neurons is neuronal pentraxin 1 (NP1) (DeGregorio-Rocasolano et al., 2001). NP1 is predominantly expressed in the nervous system (Schlimgen et al., 1995). In previous studies, we have shown that reduction of neuronal activity induces the synthesis of NP1 (DeGregorioRocasolano et al., 2001) through a glycogen synthase kinase 3 signaling pathway (Enguita et al., 2005). The increase in NP1 occurs during the initial phase of the gene-expression-dependent intrinsic program of apoptotic death, before neurons reach the irreversible commitment to die. Moreover, NP1 also contributes to synapse loss, neurite damage, and the subsequent apoptotic toxicity induced by other apoptotic stimuli such as amyloid- $\beta$ $(\mathrm{A} \beta)$ in cortical neuronal cultures (Abad et al., 2006).

It is well established that the apoptosis evoked by low neuronal activity in cerebellar granule neurons (CGNs) requires macromolecular synthesis-dependent BAX translocation to the mito-

Received Sept. 8, 2011; revised Nov. 2, 2011; accepted Nov. 23, 2011.

Author contributions: R.T. designed research; K.B.C., P.P., J.F.-S., G.L.-D., L.B., M.E., and M.A.A. performed research;P.P., J.F.-S., M.E., and E.S. contributed unpublished reagents/analytic tools; K.B.C.,P.P., G.L.-D.,M.E.,M.A.A., E.S., and R.T. analyzed data; K.B.C. and R.T. wrote the paper.

This work has been funded by Ministerio de Ciencia e Innovación of Spain Grants SAF2008-03514 (R.T.) and BFU2008-3980 (E.S.) and by the Centro de Investigación Biomédica en Red de Enfermedades Neurodegenerativas. We thank Nuria Serra and Natalia Castro for technical assistance.

*K.B.C. and P.P. contributed equally to this work.

Correspondence should be addressed to Ramon Trullas, Neurobiology Unit, Biomedical Research Institute of Barcelona, Consejo Superior de Investigaciones Científicas, Institute of Biomedical Research August Pi i Sunyer, Rosselló 161, 08036 Barcelona, Spain. E-mail: ramon.trullas@iibb.csic.es.

DOI:10.1523/JNEUROSCI.4604-11.2012

Copyright $\odot 2012$ the authors $\quad 0270-6474 / 12 / 321453-14 \$ 15.00 / 0$ chondria (Putcha et al., 1999). During activation of the intrinsic program of apoptosis, BAX translocates to mitochondria and forms large homo-oligomer complexes that permeabilize the outer mitochondrial membrane, allowing the release of pro-apoptotic factors, such as cytochrome $c$ (Youle and Strasser, 2008). However, the signal transduction pathways and mechanisms that lead to the neuronal activitydependent BAX translocation to mitochondria are not yet resolved. We hypothesized that proteins, such as NP1, that are particularly expressed in neurons and whose expression is induced by low neuronal activity might contribute to the targeting of BAX to mitochondria.

Mitochondria are dynamic organelles that divide and fuse, and it is the balance between these two complementary processes that determines the morphology of the mitochondrial network; in unhealthy cells, division (fission) becomes predominant and the mitochondrial network fragments (Suen et al., 2008). Recently, it has become evident that pro- and anti-apoptotic members of the Bcl-2 family are involved in the regulation of the mitochondrial network in both healthy and stressed cells (Autret and Martin, 2009). BAX, for example, appears to have a different physiological role in regulating mitochondrial dynamics in healthy cells that is separate from its pro-apoptotic function (Karbowski et al., 2006). A similar, but contrary, role has been reported for anti-apoptotic members of the Bcl-2 family of proteins, such as BCL- $\mathrm{x}_{\mathrm{L}}$ (Berman et al., 2009). Nonetheless, the relationship between the apoptosis program and mitochondrial dynamics is still subject to investigation.

In the present study, we have investigated the function of NP1 within the biochemical cascade of the apoptotic program evoked by low neuronal activity as well as its role in mitochondrial dynamics. We found that NP1 is upstream of BAX in the apoptotic 
program of cell death activated by low neuronal activity and that NP1 is preferentially located at mitochondria after $\mathrm{K}^{+}$deprivation. In addition, we show that NP1 colocalizes with BAX in the mitochondria and that mitochondrial fragmentation induced by low neuronal activity requires NP1 expression.

\section{Materials and Methods}

Cell culture. Primary cultures of CGNs were prepared from postnatal day 7 Sprague Dawley rat pups or from postnatal day 7 pups from heterozygous B6.129X1-Baxtm1Sjk/J BAX knock-out mice (The Jackson Laboratory) of either sex as described previously (DeGregorio-Rocasolano et al., 2001). Cells were dissociated in the presence of trypsin and DNaseI and plated in poly-L-lysine $(100 \mu \mathrm{g} / \mathrm{ml})$-coated dishes at a density of $3 \times 10^{5}$ cells $/ \mathrm{cm}^{2}$ for rat neurons and $2 \times 10^{5}$ cells $/ \mathrm{cm}^{2}$ for mouse neurons in basal Eagle's medium supplemented with $10 \%$ heat-inactivated fetal bovine serum, $0.1 \mathrm{mg} / \mathrm{ml}$ gentamicin, $2 \mathrm{~mm}$ L-glutamine, and $25 \mathrm{~mm} \mathrm{KCl}$. Cytosine-D-arabinofuranoside $(10 \mu \mathrm{M})$ was added to the culture medium $18-20 \mathrm{~h}$ after plating to prevent replication of non-neuronal cells. Cells were maintained at $37^{\circ} \mathrm{C}$ in a humidified incubator with $5 \% \mathrm{CO}_{2}$ and $95 \%$ air. The cultures were left undisturbed until 7-8 $\mathrm{d}$ in vitro (DIV) for the experiments. For mitochondrial transport experiments, primary cultures of hippocampal neurons were obtained from E15-E17 OF1 mouse embryo brains of either sex. Hippocampal neurons were seeded onto poly-L-lysine-coated Fluorodish plates (World Precision Instruments) and cultured as above. All procedures involving animals and their care were approved by the ethics committee of the University of Barcelona, and they were conducted in accordance with guidelines that conform with national (Generalitat de Catalunya) and international laws and policies (Guide for the Care and Use of Laboratory Animals).

Genotyping of mice with different gene dosages of Bax. Tail DNA was obtained following the protocol of The Jackson Laboratory. One millimeter of tail was cut and incubated at $55^{\circ} \mathrm{C}$ in a buffer containing $50 \mathrm{~mm}$ $\mathrm{KCl}, 10 \mathrm{~mm}$ Tris- $\mathrm{HCl}$, pH 8.3, $2.5 \mathrm{~mm} \mathrm{MgCl}_{2} \cdot 6 \mathrm{H}_{2} \mathrm{O}, 0.45 \%$ (v/v) Nonidet $\mathrm{P}-40,0.45 \%$ (v/v) Tween 20, and $150 \mu \mathrm{g} / \mathrm{ml}$ Proteinase K (Invitrogen). After tissue digestion, the samples were incubated for $5 \mathrm{~min}$ at $95^{\circ} \mathrm{C}$ to inactivate Proteinase K, and $2 \mu \mathrm{l}$ of this sample was used for PCR analysis. Both the wild-type and the mutant Bax alleles were screened by PCR. The wild-type allele was amplified with slightly modified primers using an exon $5^{\prime}$ forward primer (5'-GAGCTGATCAGAACCATCATG-3') and an intron 5 reverse primer (5'-GGTTGACCAGAGT GCGTAGG-3'), which generated a fragment of $312 \mathrm{bp}$. The mutant allele was amplified using a neo/pgk primer (5'-CCGCTTCCATTGCTC AGCGG-3') and the same intron 5 reverse primer. In this case, the size of the product generated was $507 \mathrm{bp}$. Cycling parameters were as follows: an initial step of $10 \mathrm{~min}$ at $95^{\circ} \mathrm{C}, 15 \mathrm{~s}$ at $95^{\circ} \mathrm{C}$ and $1 \mathrm{~min}$ at $60^{\circ} \mathrm{C}$ each for a total of 35 cycles, and after these, a final single step of $7 \mathrm{~min}$ at $72^{\circ} \mathrm{C}$. PCR products were analyzed on $2 \%$ agarose gels.

Induction of neuronal death by potassium deprivation. At 7-8 d after plating, the medium in which the cells were grown was replaced with either fresh serum-free medium supplemented with $25 \mathrm{~mm}$ potassium (high $\mathrm{K}^{+}$or $\mathrm{K}^{+}$) or fresh serum-free medium containing $5 \mathrm{~mm}$ potassium $\left(\right.$ low $\mathrm{K}^{+}$or $\mathrm{K}^{-}$).

Determination of cell viability and apoptosis in cultures. For studying cell viability, we used two different approaches. In some experiments, cell death was assessed using propidium iodide (PI) staining, which is excluded from viable cells. Damage to the plasma membrane permits the entry of PI. Inside the cell, PI interacts with nuclear DNA and yields a bright red fluorescence. This fluorescence was measured in 24-well plates using CytoFluor 2350 scanner (Millipore Corporation) with $530 \mathrm{~nm}$ excitation ( $25 \mathrm{~nm}$ bandpass) and $645 \mathrm{~nm}$ emission (40 nm bandpass) filters. The percentage of non-viable cells was measured as described previously (Enguita et al., 2005). Baseline fluorescence $\left(F_{1}\right)$ was measured $1 \mathrm{~h}$ after the addition of $30 \mu \mathrm{M}$ PI showing the death not related to the treatment. Several fluorescence readings were taken at different times. Maximum fluorescence corresponding to $100 \%$ of cell death $\left(F_{\max }\right)$ was measured after incubation at $37^{\circ} \mathrm{C}$ for $10 \mathrm{~min}$ in the presence of $500 \mu \mathrm{M}$ digitonin. The percentage of cell death was calculated following this formula: $\%$ cell death $=100 \times\left(F_{n}-F_{1}\right) /\left(F_{\max }-F_{1}\right)$, where $F_{n}$ is the fluorescence at any given time. In other experiments, cell viability was analyzed by nuclei staining with Hoechst 33258 (Sigma) for morphological determination of apoptosis. CGNs were washed with ice-cold PBS, fixed with $4 \%$ paraformaldehyde in PBS containing $2 \%$ sucrose for 30 min at room temperature (RT), and subsequently incubated with icecold methanol for $2 \mathrm{~min}$. After some washes with ice-cold PBS, cells were stained with Hoechst 33258 (175 $\mathrm{ng} / \mathrm{ml}$ in PBS) for $10 \mathrm{~min}$ at RT, and finally neurons were washed twice with PBS and covered with glycerol. Nuclei were visualized using an Olympus IX70 inverted fluorescence microscope with a $30 \times$ objective. In each experiment, photomicrographs were taken from three randomly chosen fields per well by an observer blinded to the treatments. At least three wells were examined for each treatment.

Lentiviral vectors and constructs. The self-inactivating bicistronic lentiviral transfer vector constructs pWPI, pLVTHM, and pWPXL, as well as the second-generation lentivirus packaging and envelope plasmids, were kindly provided by Dr. D. Trono (Lausanne, Switzerland). The map and the sequences of these plasmids are available at Addgene (http://www. addgene.org/). The constructs pWPI-NP1-GFP, for NP1 overexpression, pLVTHM-shRNAi-NP1-GFP, for the silencing of NP1, and pLVTHM-shRandom-GFP, with a control non-target sequence, all of them expressing GFP in a bicistronic manner, were obtained as described previously (Abad et al., 2006). The construct pWPXL-MtDsRed was obtained by inserting within the restriction sites BamH1 and EcoR1 the sequence of DsRed containing the mitochondrial targeting sequence of cytochrome $c$ oxidase complex IV (COX IV) at the N-terminal side. An NP2-NP1 hybrid construct was obtained by substituting the first 240 bases of NP1 starting at the initiation codon with the corresponding bases of neuronal pentraxin 2 (NP2). The DNA coding sequence bases $1-240$ of NP2 and 241-1299 of NP1 were amplified by PCR. The resulting fragments were annealed and reamplified using NP2 $3^{\prime}$ forward and NP1 $5^{\prime}$ reverse primers. The hybrid sequence was subcloned in the pWPXL vector within BamH1 and EcoR1 restriction sites.

Viral particles pseudotyped with the vesicular stomatitis virus G glycoprotein were produced by transient transfection in HEK 293T cells plated in $100 \mathrm{~mm}$ dishes in DMEM plus 10\% FBS. When subconfluent, HEK 293T cells were cotransfected with $20 \mu \mathrm{g}$ of lentiviral vector containing the transgene, $15 \mu \mathrm{g}$ of the packaging plasmid psPAX2, and $6 \mu \mathrm{g}$ of the envelope plasmid pMD2G using calcium phosphate. High-titer stocks $\left[1 \times 10^{7}\right.$ transduction units (TU) per microliter] were obtained by ultracentrifugation and resuspension of the viral pellet in TNE buffer (in mM: 50 Tris- $\mathrm{HCl}, \mathrm{pH} 7.5,130 \mathrm{NaCl}$, and 1 EDTA). Viral stocks were stored at $-20^{\circ} \mathrm{C}$ and used within 1 month.

Transduction of CGNs with lentiviral vectors. CGNs were transduced immediately after plating with $\sim 10 \times 10^{7} \mathrm{TU}$. This lentiviral titer was found to produce no significant cell toxicity. A lentiviral vector carrying a silencing cassette expressing an shRNA against no known target (shRandom-GFP) was used as control for knockdown experiments. An empty pWPI lentiviral vector was used as control in transgene overexpression experiments. After addition of the lentivirus, cultures were maintained at $37^{\circ} \mathrm{C}$ for $20 \mathrm{~h}$, and then the medium was changed to fresh BME plus 10\% FBS. The percentage of neurons expressing GFP $48 \mathrm{~h}$ after transduction was $70-80 \%$. In these cells, potassium deprivation experiments were performed at DIV7-8.

Live imaging and quantification of axonal transport of mitochondria. Hippocampal neurons were transfected with MtDsRed and either shRandom-GFP or shNP1-GFP and imaged $24-48 \mathrm{~h}$ later using a Leica TCS SP2 confocal microscope equipped with a $63 \times$ oil-immersion objective. Cultures were maintained at $37^{\circ} \mathrm{C}$ using a heating insert on the microscope stage and an incubating chamber, allowing circulation of a controlled heated $\mathrm{CO}_{2}(5 \%)$-air mixture for the control of $\mathrm{pH}$. For measurements of axonoplasmic transport of mitochondria, axonal processes in transfected neurons were identified by GFP expression following morphological criteria, and directionality was determined for each axon. Axonal mitochondria were registered with an additional digital zoom of $1.7 \times$. Time-lapse series of image stacks composed of five images $(512 \times$ 512 pixels) were taken every $6 \mathrm{~s}$ for $15 \mathrm{~min}$. All 151 images obtained were processed primarily with Leica confocal software. Additional image processing, analysis, and video compilation (at a frame rate of $10 / \mathrm{s}$ ) and 
editing was performed with NIH ImageJ software (version 1.43K). Kymographs were generated with MetaMorph Software (Molecular Devices/MDS Analytical Technologies). In all cases, a mitochondrion was considered motile when it moved $>0.5 \mu \mathrm{m}$ during $1 \mathrm{~min}$ of recording. Distances, speeds of retrograde, and anterograde transport were measured separately from the corresponding kymographs. To control for the number of mitochondria analyzed, the average length was calculated for each neuron and then the mean was calculated between axons.

SDS-PAGE and Western blot. Cells were solubilized in SDS sample buffer (62.5 mм Tris-HCl, pH 6.8, 2\% SDS, 10\% glycerol, 100 mм DTT, and $0.01 \%$ bromophenol blue). After harvesting the cells, the extracts were warmed at $85^{\circ} \mathrm{C}$ for $3 \mathrm{~min}$ and then briefly sonicated. The polypeptides were separated by SDS-PAGE. NP1 was assayed in either 10 or $12 \%$ SDS-polyacrylamide gels. BAX, BIM $\mathrm{EL}_{\mathrm{EL}}$, cleaved caspase-3, and cytochrome $c$ were analyzed in $15 \%$ gels. After electrophoresis, the samples were electroblotted onto polyvinylidene difluoride (PVDF) membranes (Millipore) according to the protocol of the manufacturer. Blots were blocked with 5\% nonfat dry milk (NFM) in Tris-buffered saline containing $0.1 \%$ Tween (TBS-T) before immunodetection. All primary antibodies were prepared at the appropriate dilution in TBS-T solution containing 3\% bovine serum albumin (Sigma). The antibodies used included the following: mouse monoclonal anti-rat NP1 (1:1000; BD Biosciences), rabbit anti-cleaved caspase-3 (Asp 175) (1:750; Cell Signaling Technology), mouse monoclonal anti-cytochrome $c$ (1:1000; BD Biosciences), rabbit anti-BAX (N-terminal) (1:1000; Millipore), and rabbit anti-BIM (C34C5) (1:500; Cell Signaling Technology). A rabbit antiactin antibody (1:3000; Sigma) was used as a loading control for total and cytosol-enriched fractions. To control for the amount of protein loaded in mitochondria-enriched fractions, we used a monoclonal anti-COX IV antibody (1:5000; Invitrogen). Peroxidase-conjugated goat anti-mouse IgG (1:10,000; Jackson ImmunoResearch) or peroxidase-conjugated goat anti-rabbit IgG (1:5000; Cell Signaling Technology) were the secondary antibodies used in a 5\% NFM solution in TBS-T. Immunoreactive proteins were visualized using an enhanced chemiluminescence detection system (SuperSignal West Dura; Pierce), and the detection of the bands was performed using a VersaDoc Model 5000 Imaging System quantifying their intensity with the Quantity One computer software (Bio-Rad). The densitometry of the bands corresponding to total or cytosolic NP1, cleaved caspase-3, or cytosolic BAX was normalized to the corresponding actin analysis. The values of cytochrome $c, \mathrm{BAX}$, and NP1 bands in mitochondria-enriched fractions were normalized to the densitometry of the corresponding COX IV band.

Measurement of cell-surface levels of NP1. CGNs were biotinylated using the Cell Surface Protein Isolation kit (Pierce) following the protocol of the manufacturer. Cells were washed twice with cold PBS and incubated at $4^{\circ} \mathrm{C}$ with biotinylation reagent $(0.25 \mathrm{mg} / \mathrm{ml}$ sulfo-NHS-SS-Biotin; Pierce) for 30 min with gentle agitation. The biotinylation reaction was stopped by addition of cold quenching solution (Pierce). Cells were lysed, and solubilized proteins were incubated for $1 \mathrm{~h}$ at RT with Immobilized NeutrAvidinTM Gel slurry (Pierce). The bound biotinylated proteins were eluted by incubating for $1 \mathrm{~h}$ at RT with SDS-PAGE buffer $(62.5$ mм Tris-HCl, pH 6.8, 1\% SDS, 10\% glycerol, and 50 mм DTT), followed by centrifugation for $2 \mathrm{~min}$ at $1000 \times \mathrm{g}$. Biotinylated proteins were resolved by $8 \%$ SDS-PAGE and transferred to PVDF membranes (Millipore). We used actin as a loading control for total protein extract and as a standard control to ensure that there was no biotinylation of intracellular proteins. The labeling of proteins was visualized by ECL and quantified as described above. The proportion of NP1 at the cell surface was determined by measuring the band densities of biotinylated NP1 and the NP1 in the total lysate.

Subcellular fractionation. Subcellular fractionation was performed in cultured rat CGNs plated in six-well plates. In the experiments investigating gain or loss of NP1 function, cells were transduced with the lentiviral vector for transgene overexpression of NP1 (pWPI-NP1) or with the vector for NP1 silencing (shNP1) at DIV0. Treatment with low $\mathrm{K}^{+}$ was performed when cells were mature at DIV7-8. At different times after treatment with low $\mathrm{K}^{+}$, cells were harvested, and mitochondriaenriched fractions were obtained using the Mitochondrial Isolation Kit for Cultured Cells from Pierce Thermo Scientific following the instruc- tions of the manufacturer. Mitochondria-enriched pellets were solubilized in SDS sample buffer and warmed $3 \mathrm{~min}$ at $85^{\circ} \mathrm{C}$. Fractions were analyzed for cytochrome $c, \mathrm{BAX}$, and NP1 by Western blot as described above.

Immunocytochemistry. CGNs were plated at a density of $2 \times 10^{5}$ cells/ $\mathrm{cm}^{2}$ on chamber slides (LabTek, VWR) or glass coverslips coated with poly-L-lysine $(100 \mu \mathrm{g} / \mathrm{ml})$. After plating, the cells were transduced with pWPXL-MtDsRed and the corresponding lentiviral vectors as described above. Cells were treated with high or low $\mathrm{K}^{+}$at DIV7-8. After 4-8 h, the medium was removed, and the cells were rinsed in PBS and fixed in $4 \%$ paraformaldehyde in PBS containing $2 \%$ sucrose at $4^{\circ} \mathrm{C}$ for $10 \mathrm{~min}$. After 5 min incubation with $0.1 \mathrm{M}$ glycine/PBS, cells were permeabilized in $0.5 \%$ CHAPS/PBS for 30 min before washing briefly in PBS. Blocking was performed in $5 \% \mathrm{NGS} / \mathrm{PBS}$ for $30 \mathrm{~min}$ at RT. Incubation with primary antibodies, diluted in $1 \% \mathrm{NGS} / \mathrm{PBS}$, was performed either overnight at $4^{\circ} \mathrm{C}$ or for $3 \mathrm{~h}$ at RT. Primary antibodies used were mouse anti-NP1 (1:50; BD Biosciences), rabbit anti-NP1 (1:100; BD Biosciences), and/or mouse anti-BAX clone 6A7 (1:100; BD Biosciences). After incubation, cells were washed in 1\% NGS/PBS and incubated in secondary antibodies, Alexa Fluor-488 anti-mouse/rabbit or Alexa Fluor-633 anti-mouse (Invitrogen) at 1:1000 for $1 \mathrm{~h}$ at RT. After PBS washes, cells were coverslipped using Prolong Gold Antifade (Invitrogen). In some cases, CGNs were coverslipped after fixing without performing immunocytochemistry for the analysis of mitochondria marked with MtDsRed.

Imaging and analysis. Images were acquired using a $63 \times$ oilimmersion objective (numerical aperture 1.4) on a Leica TCS SPE confocal microscope. Each channel was scanned separately using identical settings for laser power and photomultiplier gain/offset within each experiment. No crosstalk or bleed-through between different channels occurred, and no appreciable signal was visible in secondary antibody controls. For determining the percentage of active BAX-positive cells, single-slice images were taken. For imaging of the mitochondrial network (as marked by MtDsRed), stacks were taken using $0.5 \mu \mathrm{m}$ or system optimized step sizes. Maximum projections were obtained using NIH ImageJ, and mitochondrial length was determined by selecting neuritic regions and analyzing the lengths of thresholded mitochondria using the NIH ImageJ "Analyze Particles" module. In a similar manner, the areas occupied by NP1 and mitochondria were quantified to show the effect of deprivation on NP1 levels at neuritic mitochondria. The length of neuronal processes was measured using NIH ImageJ software. Neuritic mitochondrial mass was calculated by multiplying the mean mitochondrial length by the number of mitochondria divided by neurite length. Volume rendering of NP1 and MtDsRed was performed using the VolumeJ plug-in for NIH ImageJ. For orthogonal sectioning, stacks were obtained using the optimal step size as determined by the Leica confocal software. Line plot analysis was performed (using the MtDsRed signal as a guide) in single optical slices with the Profile Plot function of NIH ImageJ. Image adjustment was performed after analysis for display purposes only.

Immunoprecipitation. BAX immunoprecipitation was performed in protein extracts from either total brain or CGNs treated with low $\mathrm{K}^{+}$to assess the interaction of native proteins or from HEK 293T cells transfected with recombinant rat NP1 and BAX cDNAs. For total rat brain extraction, the whole rat brain was Dounce homogenized in $10 \mathrm{vol}$ of immunoprecipitation buffer (in mM: 50 Tris- $\mathrm{HCl}, \mathrm{pH} 7.5,100 \mathrm{NaCl}$, and $2 \mathrm{CaCl}_{2}$ ) containing $1 \%$ Triton X-100 and mini-EDTA Free protease inhibitor cocktail (Roche). The lysate was centrifuged at $1000 \times g$ for 5 min, and the supernatant was incubated $1 \mathrm{~h}$ at $4^{\circ} \mathrm{C}$ and centrifuged at $50,000 \times g$ for $15 \mathrm{~min}$. CGNs were potassium deprived for $2 \mathrm{~h}$ on DIV7-8, washed twice with PBS on ice, and lysed in immunoprecipitation buffer (as for total brain). The lysate was incubated for 20 min on ice and centrifuged at $4^{\circ} \mathrm{C}$ at $10,000 \times g$ for $10 \mathrm{~min}$ to remove insoluble components. HEK $293 \mathrm{~T}$ cells were transfected using a standard calcium phosphate transfection protocol. At $24 \mathrm{~h}$ after transfection, the cells were washed twice with PBS on ice and lysed in immunoprecipitation buffer containing 1\% Triton X-100 and mini-EDTA Free protease inhibitor cocktail (Roche); supernatants were obtained as for CGNs. The immunoprecipitation of the supernatants-total brain, CGNs, and HEK 
293Ts — was performed using Protein A Magnetic Beads (New England Biolabs) following the instructions of the manufacturer. Briefly, the lysate was incubated for $20 \mathrm{~min}$ at RT with the magnetic beads previously bound to $4 \mu \mathrm{g}$ of the rabbit anti-BaxNT antibody (Millipore). For the control immunoprecipitations, we used $4 \mu \mathrm{g}$ of ChromePure rabbit IgG whole molecule (Jackson ImmunoResearch). After immunoprecipitation, the beads were washed three times with PBS, eluted in SDS sample buffer for $5 \mathrm{~min}$ at $85^{\circ} \mathrm{C}$, and subjected to Western blotting with the corresponding antibodies.

Statistical analyses. The results are expressed as the mean \pm SEM. Unless otherwise specified, the $n$ value refers to the number of independent cell cultures. The statistical significance of the differences was examined using independent $t$ tests or one-way ANOVA with Bonferroni's post hoc multiple comparisons tests when required.

\section{Results}

\section{Treatment of CGNs with low $\mathrm{K}^{+}$increases the amount of NP1} in mitochondria

We have shown previously that NP1 overexpression precedes cell death induced by $\mathrm{K}^{+}$deprivation in cultured CGNs and that this increase in NP1 expression contributes to apoptosis (DeGregorio-Rocasolano et al., 2001; Enguita et al., 2005). To investigate the mechanisms of this pro-apoptotic effect, we analyzed the subcellular localization of NP1 after induction of the apoptotic program by low $\mathrm{K}^{+}$. First, we biotinylated the proteins located at the neuronal surface to determine the amount of NP1 tethered to the neuronal membrane with exposed residues at the extracellular space. The results showed that the amount of NP1 at the neuronal surface in control CGNs is a small fraction $(5 \pm 1 \%)$ of the total cellular NP1 (Fig. $1 A$ ). In agreement with previous results, treatment with low $\mathrm{K}^{+}$caused a marked increase $(310 \pm 26 \%)$ in total NP1 but did not significantly modify the amount of NP1 at the cytoplasmic membrane surface $(8 \pm 2 \%)$, indicating that the amount of NP1 evoked by low $\mathrm{K}^{+}$was either released or targeted to other locations (Fig. $1 A)$. Because we could not detect secreted NP1 in the culture medium either before or after $\mathrm{K}^{+}$deprivation (data not shown), we next performed biochemical experiments with subcellular fractions to determine the subcellular distribution of the NP1 evoked by low $\mathrm{K}^{+}$. NP1 was not detected in nuclear fractions (results not shown). In contrast, as shown in Figure $1 B$, treatment of CGNs with low $\mathrm{K}^{+}$caused a significant increase in protein levels of NP1 in mitochondria-enriched fractions (282 \pm $45 \%$ ) when compared with high $\mathrm{K}^{+}$control cells. In comparison, low $\mathrm{K}^{+}$caused a small increase in NP1 in cytosolic fractions $(180 \pm 24 \%)$, but this effect did not reach statistical significance (Fig. $1 B$ ). These results suggest that the NP1 induced after activation of the apoptotic program by low $\mathrm{K}^{+}$is located predominantly at mitochondria.

To substantiate further the subcellular localization of NP1, we performed immunocytochemical studies in CGNs that were transduced with MtDsRed, a red fluorescent protein targeted to mitochondria. Confocal images of MtDsRed and NP1 immunofluorescence showed that treatment with low $\mathrm{K}^{+}$caused a marked increase in NP1 immunofluorescence in mitochondrial regions (Fig. 1C,D). Subsequent quantitative analyses of line profile plots performed on images of equivalent MtDsRed fluorescence between treated and control cells confirmed that treatment of CGNs with low $\mathrm{K}^{+}$caused a significant increase in NP1 immunofluorescence in mitochondria $(314 \pm 31 \%$ over that in high $\mathrm{K}^{+}$controls) (Fig. $1 E$ ). Together, these findings confirm the results observed in the biochemical subcellular fractionation studies and indicate that the NP1 synthesized after activation of the apoptotic program by low $\mathrm{K}^{+}$is predominantly targeted to mitochondria.

To explore further the targeting of NP1 to mitochondria, we examined the influence of the N-terminal side of NP1, which contains a putative mitochondrial targeting sequence, on its subcellular distribution. We expressed in HEK 293T cells, an NP2NP1 hybrid protein in which the first 80 amino acids of the NP1 $\mathrm{N}$ terminus were substituted with the corresponding amino acids of NP2, another protein of the same family that contains a functional signal peptide (Tsui et al., 1996). The subcellular distribution of the hybrid NP2-NP1 protein was significantly different from that of NP1. Compared with NP1, the amount of the hybrid NP2-NP1 was lower $(50 \pm 5 \%)$ in the mitochondrial enriched fraction and higher $(278 \pm 31 \%)$ in the cytosolic fraction, indicating that the first 80 amino acids of NP1 N-terminal side contribute to its mitochondrial localization (results not shown).

\section{Low $\mathrm{K}^{+}$induces mitochondrial fragmentation associated with NP1 at neuritic mitochondria}

Because disruption of the mitochondrial network has been linked to the apoptotic cascade, we decided to study the role of NP1 in mitochondrial fragmentation. Reduction of extracellular $\mathrm{K}^{+}$significantly decreased mean neuritic mitochondrial length by $\sim 33 \%$ (from $2.1 \pm 0.1 \mu \mathrm{m}$ in controls to $1.4 \pm 0.2 \mu \mathrm{m}$ after $6 \mathrm{~h}$ of treatment with low $\mathrm{K}^{+}$) (Fig. $2 A, B$ ). Although analyses of mitochondrial length were performed specifically in the neurites, fragmented mitochondria were evident in the soma as well (Fig. $2 B$ ). In agreement with previous work (Loucks et al., 2009), fragmentation was detectable after 6 and $8 \mathrm{~h}$ of $\mathrm{K}^{+}$deprivation but not at $4 \mathrm{~h}$, whereas mitochondrial length in control cultures maintained in high $\mathrm{K}^{+}$was not significantly altered over the time course (Fig. 2A). Next, we performed confocal imaging experiments on CGNs that had been $\mathrm{K}^{+}$deprived for 4, 6, and $8 \mathrm{~h}$ (Fig. $2 C, D$ ) and measured the amount of NP1 puncta colocalizing with mitochondria located in neurites. To account for differences in mitochondrial area, we expressed this value as the percentage of MtDsRed signal. Although NP1 puncta were also clearly visible in control neurites, a small but significant increase in NP1 protein in neurites near mitochondria was evident by $4 \mathrm{~h}$ of $\mathrm{K}^{+}$deprivation (from $10 \pm 1 \%$ in controls to $17 \pm 3 \%$ in low $\mathrm{K}^{+}$). The associations were increased further and reached a maximum after 6 of $\mathrm{K}^{+}$deprivation (to $26 \pm 3 \%$ ) (Fig. 2C). Furthermore, using higher-resolution confocal imaging, NP1 puncta could be found at mitochondrial tips and putative scission sites, at times encircling thinner regions within thicker mitochondria (Fig. 2E, arrows).

\section{NP1 is required for mitochondrial fragmentation in $\mathrm{K}^{+}$-deprived CGNs}

Because of the close physical and temporal association between NP1 and fragmented mitochondria, we next studied what effect knockdown of NP1 has on low $\mathrm{K}^{+}$-induced mitochondrial fragmentation. NP1 knockdown was performed using RNAi via transduction of the silencing vector pLVTHM-shRNAi-NP1 (shNP1). As shown in Figure $3 A$, transduction of CGNs with shNP1 reduced basal NP1 protein levels and completely blocked the increase in NP1 induced by low $\mathrm{K}^{+}$. Thus, low $\mathrm{K}^{+}$increased $\mathrm{NP} 1$ levels to $299 \pm 51 \%$ of high $\mathrm{K}^{+}$controls; this was reduced to $91 \pm 21 \%$ of control in low $\mathrm{K}^{+}$-treated cells transduced with shNP1. Moreover, treatment with low $\mathrm{K}^{+}$for $6-8 \mathrm{~h}$ caused a significant $30 \%$ reduction in mitochondrial length (from $1.9 \pm$ $0.1 \mu \mathrm{m}$ in control to $1.3 \pm 0.1 \mu \mathrm{m}$ in low $\mathrm{K}^{+}$-treated CGNs) (Fig. $3 C)$, confirming the time-course studies of the effect of potas- 

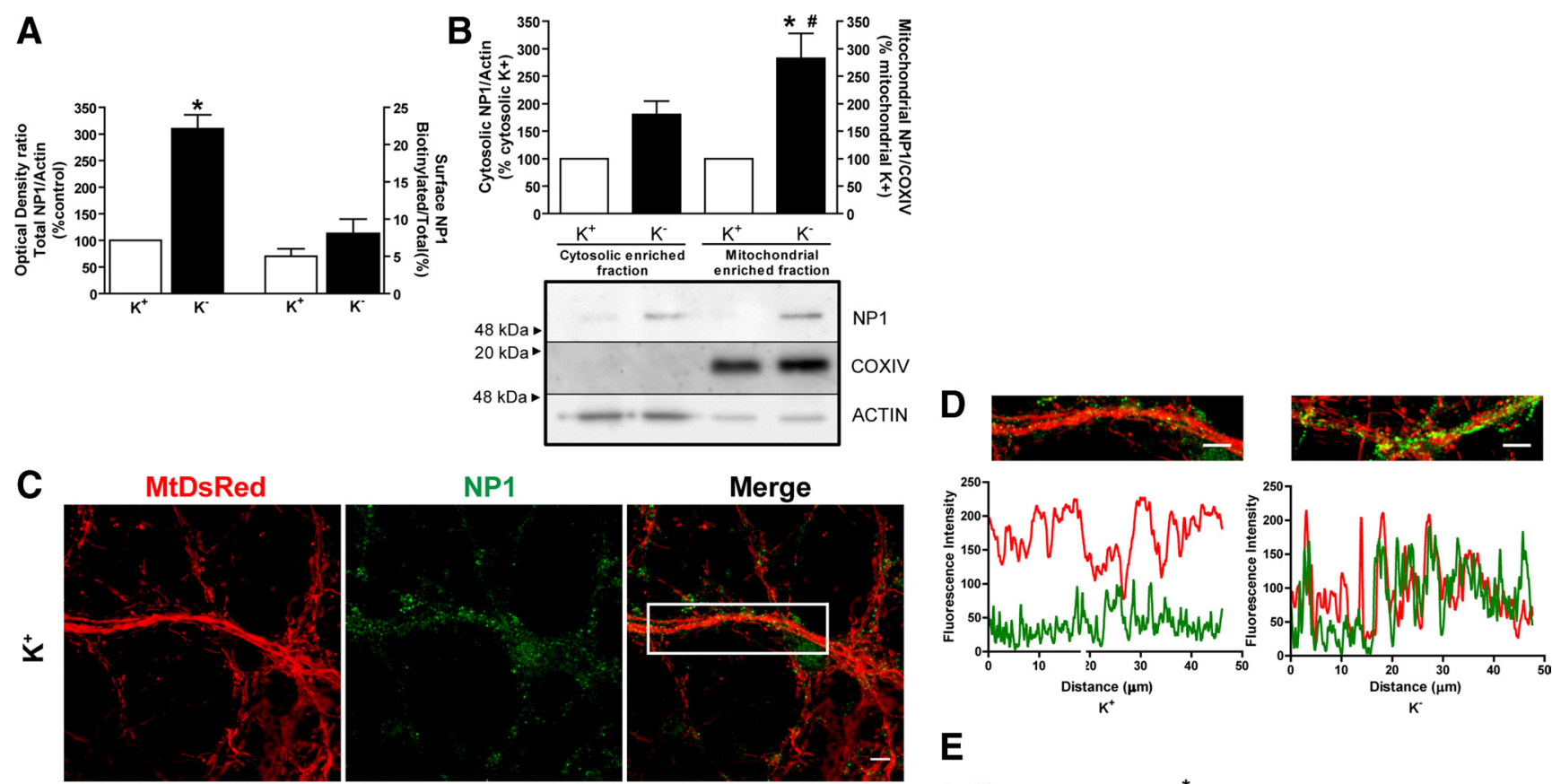

$\mathbf{E}$
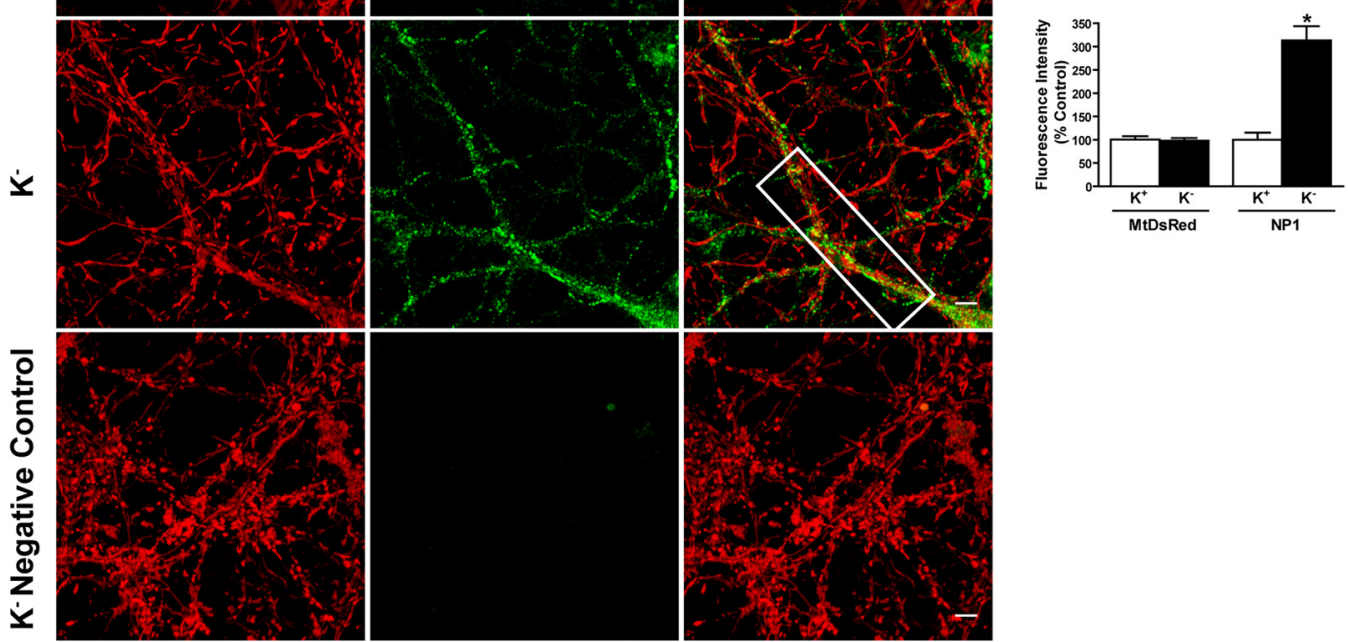

Figure 1. NP1 is preferentially located in mitochondria after low $\mathrm{K}^{+} . \boldsymbol{A}$, Quantitative analysis of surface and total NP1 after low $\mathrm{K}^{+}$treatment. At DIV7, CGNs cultures were treated with $\mathrm{K}^{+}$ deprivation for $6 \mathrm{~h}$. Then neurons were biotinylated for $30 \mathrm{~min}$ at $4^{\circ} \mathrm{C}$ to label surface proteins and NP1 levels were analyzed by Western blot and densitometric analysis. Total NP1 was normalized to the levels of actin. A marked increase in total, but no significant differences in the levels of surface, NP1 was found between $\mathrm{K}^{+}$and $\mathrm{K}^{-}$treatments. Values are mean \pm SEM. The ratio of NP1 over actin was expressed as percentage of $\mathrm{K}^{+}$values. ${ }^{*} p<0.01$, significantly different from corresponding high $\mathrm{K}^{+}$control (two-tailed independent $t$ test, $n=3$ ). $\boldsymbol{B}$, Quantification and representative Western blot of subcellular distribution of NP1 after $8 \mathrm{~h}$ of treatment with low $\mathrm{K}^{+}$. The densitometric values of NP1 bands in cytosol or mitochondria-enriched fractions were normalized to the corresponding actin or COX IV bands, respectively. Values are mean \pm SEM. ${ }^{*} p<0.001$, significantly different from corresponding high $\mathrm{K}^{+}$; ${ }^{\#} p<0.05$, significantly different from low $\mathrm{K}^{+}$ cytosol-enriched fraction (repeated-measures ANOVA with Bonferroni's multiple comparison test, $n=6$ ). $C$, Representative confocal images of mitochondria labeled with MtDsRed (red) and immunocytochemistry of NP1 (green) after $6 \mathrm{~h}$ of low $\mathrm{K}^{+}$treatment. The negative control for NP1 immunofluorescence was performed by omitting NP1 primary antibody in cells treated with low $\mathrm{K}^{+}$, when NP1 levels are high and there is mitochondrial fragmentation. Low $\mathrm{K}^{+}$treatment increased NP1 immunofluorescence in mitochondrial regions. Scale bar, $5 \mu \mathrm{m} . \boldsymbol{D}$, Higher magnification of areas marked with a white rectangle in Cand corresponding line profile plots showing increased localization of NP1 in mitochondria after treatment with low K ${ }^{+}$. Scale bar, $5 \mu \mathrm{m}$. E, Quantitative analyses of line profile plots of images with equivalent MtDsRed immunofluorescence. ${ }^{*} p<0.001$, significantly different from corresponding high $\mathrm{K}^{+}$control (two-tailed independent $t$ test, $n \geq$ 17 neurons).

sium deprivation on mitochondrial length reported previously in Figure $2 \mathrm{~A}$. Notably, knockdown of NP1 resulted in a clear blockage of low $\mathrm{K}^{+}$-induced mitochondrial fragmentation (Fig. 3C). In addition, NP1 knockdown blocked cell death induced by low $\mathrm{K}^{+}$(Fig. 3B). Thus, low $\mathrm{K}^{+}$increased cell death from $17 \pm 4 \%$ (high $\mathrm{K}^{+}$) to $69 \pm 11 \%$, whereas shNP1 transduction reduced cell death induced by low $\mathrm{K}^{+}$to $26 \pm 3 \%$. Furthermore, NP1 overexpression potentiated low $\mathrm{K}^{+}$-evoked cell death (see Fig. 6A). These results indicate that NP1 is required for mitochondrial fragmentation in response to reduced activity and suggest that NP1 regulates mitochondrial dynamics in addition to apoptosis.

\section{NP1 is involved in mitochondrial trafficking}

Mitochondrial dynamics and trafficking are essential to supply appropriate energy to distal neuronal branches and for correct neurotransmission and neuronal viability. Thus, given that NP1 is required for mitochondrial fragmentation evoked by low activity, we investigated whether NP1 is also involved in mitochondrial trafficking. We used hippocampal cultured neurons because 
they exhibit longer processes in which mitochondria can be readily identified and traced by live imaging. Neurons were transfected at DIV4 with MtDsRed and pLVTHM vectors expressing control, scrambled, or shNP1 sequences and mitochondrial motility was evaluated with live-imaging techniques. At 2-3 d after transfection, axons were identified morphologically and imaged over 15 min periods. Control neurons exhibited high rates of mitochondrial motility, which were transported in both the anterograde and retrograde directions (Fig. 4A). Only a fraction of mitochondria ( $30 \%)$ (Fig. $4 B$ ) moved over the 15 min recording periods, in agreement with previous studies (Macaskill et al., 2009). Examination of the series of images suggested that mitochondrial transport increased after knockdown of NP1. To substantiate further this notion, images were represented as kymographs (Miller and Sheetz, 2004), and mitochondrial motility was analyzed quantitatively (Fig. 4B). Comparison of mitochondrial motility in control and shNP1-transfected neurons revealed that knockdown of NP1 results in a significant increase in the percentage of moving mitochondria. Additional analysis of this effect showed that knockdown of NP1 increases the percentage of anterograde moving mitochondria without significantly altering the number of retrograde moving mitochondria. However, knockdown of NP1 did not have a significant effect on either anterograde or retrograde mitochondrial transport velocity (Fig. $4 C)$. Together, these experiments indicate that NP1, in addition to regulating mitochondrial dynamics during apoptosis, also regulates mitochondrial motility and transport in healthy living neurons.

Pro-apoptotic effect of NP1 depends on the presence of $\mathrm{BAX}$

To gain additional insight into the mechanism of the mitochondrial effects of NP1, we next measured neuronal death evoked by low $\mathrm{K}^{+}$in cultured mature CGNs from wildtype $\left(B a x^{+/+}\right)$, heterozygous $\left(\mathrm{Bax}^{+/-}\right)$, and homozygous Bax-deficient $\left(\mathrm{Bax}^{-/-}\right)$mice (Fig. $5 A$ ). In agreement with previous studies (Miller et al., 1997), exposure to reduced extracellular $\mathrm{K}^{+}$caused significant death in CGNs from both wild-type and heterozygous $B a x^{+/-}$mice but not in $B a x^{-/-}$mice. The lack of effect of low $\mathrm{K}^{+}$in cultures of $B a x^{-/-}$mice was sustained for up to $72 \mathrm{~h}$, indicating that any of the cell death pathways activated by reduction of neuronal activity requires the presence of BAX to proceed with the execution phase of the apoptotic program (Fig. 5A).

To ascertain the place of NP1 overexpression within the sequence of events of the apoptotic program induced by the reduc-

A

C

E

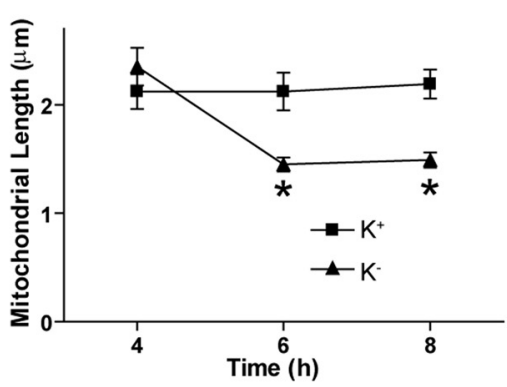

Control $\mathrm{K}^{+}$
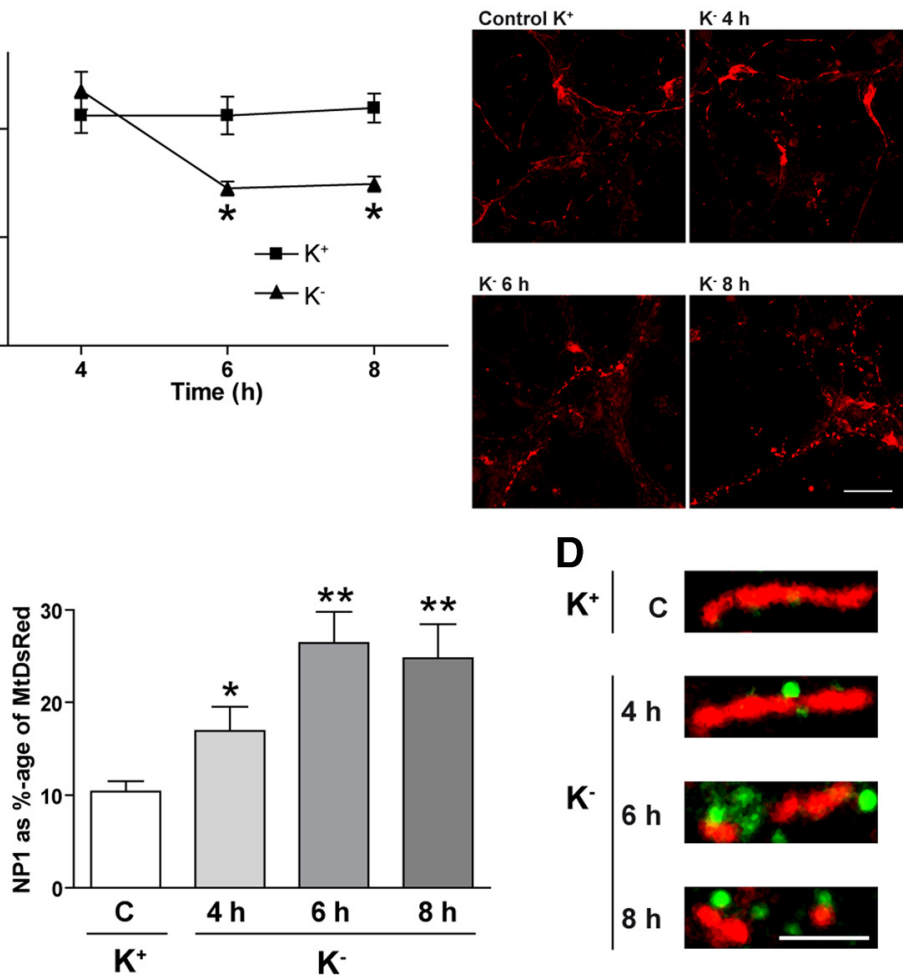

D

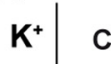

C
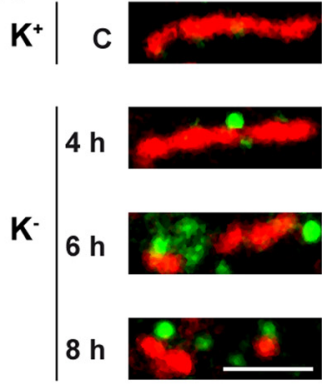

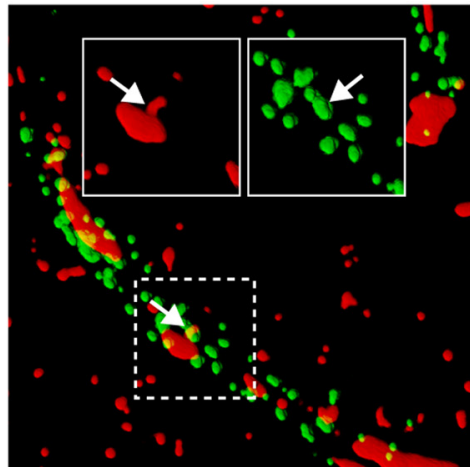

NP1 MtDsRed

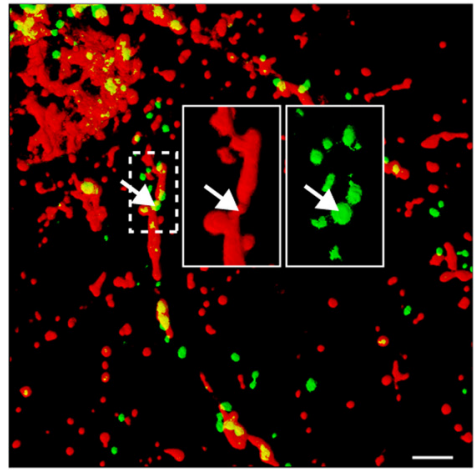

NP1 MtDsRed
Figure 2. Low $\mathrm{K}^{+}$induces mitochondrial fragmentation in CGNs in culture with a concomitant increase in mitochondrially associated NP1. Rat CGNs were transduced with MtDsRed and treated with low $\mathrm{K}^{+}$for various times before fixation and analysis. $A$, Measurement of mitochondrial length in response to low extracellular $\mathrm{K}^{+}$. Treatment with low $\mathrm{K}^{+}$resulted in a significant decrease in mitochondrial length after 6 and $8 \mathrm{~h}$, but not $4 \mathrm{~h}$, of deprivation. Values are mean \pm SEM. ${ }^{*} p<0.001$, significantly different from high $\mathrm{K}^{+}$control cultures (two-tailed independent $t$ test, $n=3$ independent cultures, $250-300$ mitochondria at each time/treatment period). $\boldsymbol{B}$, Representative images showing the mitochondrial network in control and deprived cells. Scale bar, $20 \mu \mathrm{m}$. C, NP1 levels at neuritic mitochondria after low $\mathrm{K}^{+}$treatment. Analysis was performed by expressing the area of NP1 at mitochondria in neurites as a percentage of the area occupied by mitochondria (MtDsRed). An increase in NP1 levels relative to MtDsRed was visible after 4,6 , and $8 \mathrm{~h}$. Values are mean \pm SEM. ${ }^{*} p<0.01,{ }^{* *} p<0.001$, significantly different from high $\mathrm{K}^{+}$ control cultures (two-tailed independent $t$ test, $n=4$ independent cultures, $100-200$ neurites at each time/treatment period). $\boldsymbol{D}$, Representative images showing NP1 at neuritic mitochondria over time. Scale bar, $2 \mu \mathrm{m}$. $\boldsymbol{E}$, Representative images showing NP1 in deprived CGNs in culture. Volume-rendered images showing the localization of NP1 at mitochondrial locations, including putative mitochondrial scission sites (see arrow and inset). Scale bar, $2 \mu \mathrm{m}$.

tion of neuronal activity, we studied the effect of low $\mathrm{K}^{+}$on NP1 expression in neurons from Bax-deficient mice. Treatment with low $\mathrm{K}^{+}$increased NP1 levels similarly in cultured CGNs from mice with different gene dosages of $B a x$ (Fig. $5 B$ ), indicating that NP1 overexpression evoked by low $\mathrm{K}^{+}$does not depend on the presence of BAX and that the function of NP1 is upstream or in parallel with BAX function. We next transduced CGNs from Bax- 


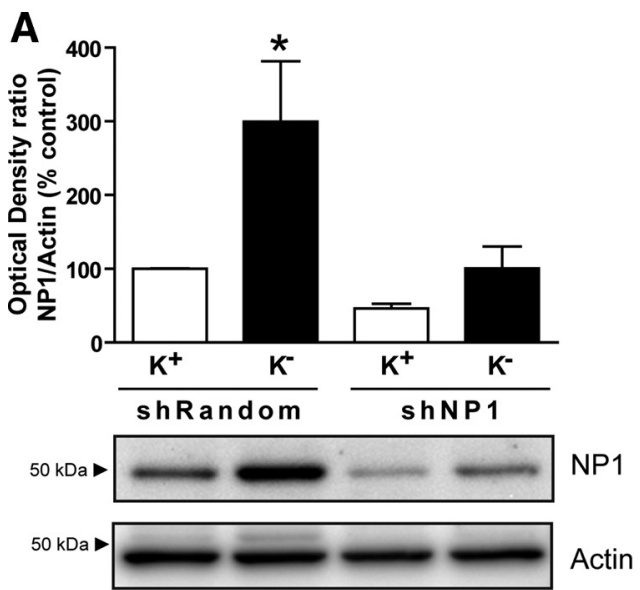

B
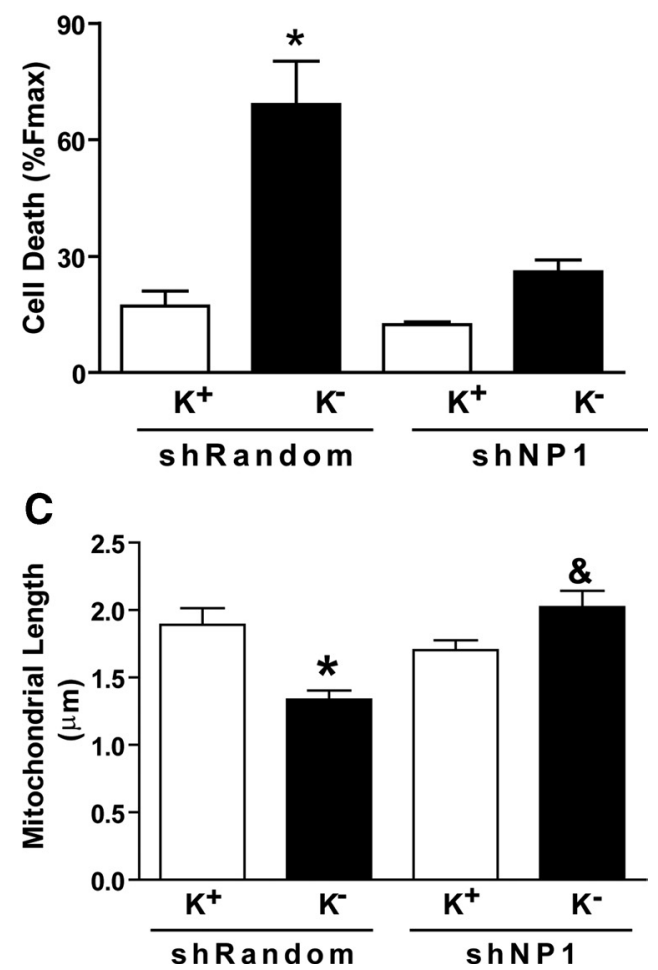

Figure 3. NP1 is required for apoptosis and mitochondrial fragmentation. CGNs were transduced with pLVTHM-shNP1 (shNP1) to knockdown NP1 or with a pLVTHM-shRandom expressing an shRNA against no known target (shRandom) and treated with low $\mathrm{K}^{+}$for $6-8 \mathrm{~h}$. $A$, Representative Western blot and quantification of NP1 levels after low $\mathrm{K}^{+}$. NP1 was normalized to the levels of actin and analyzed by densitometric analysis. Values, expressed as percentage of control, are mean \pm SEM. ${ }^{*} p<0.01$, significantly different from high $\mathrm{K}^{+} /$shRandom (one-way ANOVA with Bonferroni's multiple comparison test, $n=6$ ). $B$, Quantification of apoptosis in cultures treated with low $\mathrm{K}^{+}$. Cell death was assessed using PI staining after $24 \mathrm{~h}$ in low $\mathrm{K}^{+}$. Values are mean \pm SEM. ${ }^{*} p<0.001$, significantly different from high $\mathrm{K}^{+} /$shRandom (one-way ANOVA with Bonferroni's multiple comparison test, $n=6$ ). C, Quantification of the effect of NP1 knockdown on mitochondrial length with and without low $\mathrm{K}^{+}$. For these experiments, CGNs were transduced with MtDsRed and either shRandom or shNP1 and on DIV7- 8 were treated with low $\mathrm{K}^{+}$for $6-8 \mathrm{~h}$. Treatment with low $\mathrm{K}^{+}$significantly decreased mitochondrial length in cells transduced with the control vector but not in those transduced with shNP1. Values are mean \pm SEM. ${ }^{*} p<0.001$, significantly different from high $\mathrm{K}^{+} /$shRandom; ${ }^{\&} p<0.001$, significantly different from low $\mathrm{K}^{+} /$shRandom (two-tailed independent $t$ test, $n=2$ independent cultures, $250-400$ mitochondria at each treatment).

deficient mice with a lentiviral vector for transgene overexpression of NP1 (pWPI-NP1). In neurons from wild-type mice, NP1 overexpression significantly increased the number of apoptotic nuclei compared with cells transduced with the control vector
pWPI-Ctrl (from $15 \pm 2 \%$ to $31 \pm 3 \%$ ) (Fig. 5 C). NP1 caused a similar increase in apoptotic nuclei in neurons from $\mathrm{Bax}^{+/-}$mice (from $25 \pm 5$ to $45 \pm 9 \%$ ). In contrast, transgene overexpression of NP1 in $\mathrm{Bax}^{-1-}$ mice did not modify the number of apoptotic nuclei compared with control transduced cells (from $7 \pm 1$ to $8 \pm$ 1\%) (Fig. 5C). These results indicate that NP1 needs BAX to induce cell death and confirm that NP1 is upstream of BAX in the apoptotic program.

NP1 causes apoptosis by activating mechanisms of the intrinsic program of cell death downstream of $\mathrm{BAX}$

Following from the observation that transgene overexpression of NP1 is sufficient to induce apoptosis, we next investigated the influence of exogenous NP1 on neuronal death evoked by $\mathrm{K}^{+}$ deprivation in rat CGNs. Consistent with the results obtained in CGNs from wild-type and heterozygous Bax mice (Fig. 5C), transgene overexpression of NP1 caused a significant increase in the number of apoptotic nuclei (from $13 \pm 3 \%$ in high $\mathrm{K}^{+}$/ pWPI-Ctrl to $30 \pm 4 \%$ in high $\mathrm{K}^{+} / \mathrm{pWPI}-\mathrm{NP} 1$ ) in control untreated neurons (Fig. 6A). Moreover, NP1 significantly potentiated the increase in the number of apoptotic nuclei caused by low $\mathrm{K}^{+}\left(83 \pm 2 \%\right.$ of apoptotic nuclei in low $\mathrm{K}^{+} / \mathrm{pWPI}-\mathrm{NP} 1-$ transduced cells compared with $68 \pm 2 \%$ of apoptotic nuclei in low $\mathrm{K}^{+} /$pWPI-Ctrl-transduced neurons) (Fig. $6 \mathrm{~A}$ ).

Next, we investigated whether NP1 activates mechanisms of the apoptotic program that are downstream of BAX. It is well known that activation of BAX induces cytochrome $c$ release to the cytosol and caspase-3 cleavage (Galluzzi et al., 2009). First, we studied the effects of NP1 on cytochrome $c$ release by measuring the amount of cytochrome $c$ in mitochondria-enriched subcellular fractions. Treatment of CGNs with low $\mathrm{K}^{+}$for $8 \mathrm{~h}$ decreased the amount of cytochrome $c$ in mitochondria-enriched fractions to $53 \pm 5 \%$ of control values (Fig. $6 B$ ). Likewise, overexpression of NP1 significantly reduced (to $71 \pm 10 \%$ of control values) the amount of cytochrome $c$ in mitochondria compared with control high $\mathrm{K}^{+}$-treated cultures. This effect of NP1 was not significantly different from that observed after treatment with low $\mathrm{K}^{+}$. In addition, NP1 overexpression markedly potentiated the low $\mathrm{K}^{+}$evoked decrease in the amount of mitochondrial cytochrome $c$ (to $19 \pm 4 \%$ of control values) (Fig. $6 \mathrm{~B}$ ).

Subsequently, we studied the effect of NP1 on the cleavage of caspase-3. Western blot analysis showed that NP1 significantly potentiates the amount of cleaved caspase- 3 evoked by low $\mathrm{K}^{+}$ (Fig. 6C). This effect of NP1 was observed between 6 and $8 \mathrm{~h}$ after low $\mathrm{K}^{+}$treatment and was significantly attenuated by reducing NP1 protein levels via the simultaneous transduction of the silencing vector pLVTHM-shRNAi-NP1 (shNP1). NP1 caused small increases in the levels of cleaved caspase- 3 in control cultures in some experiments, but this effect did not reach statistical significance, probably because of the relatively small magnitude of the effect and the variability of the Western blot detection procedure. Altogether, these results indicate that NP1 overexpression is sufficient to activate mechanisms of the apoptotic program that are downstream of the activation of BAX, although NP1 requires the presence of other pro-apoptotic proteins of the intrinsic program to induce a complete apoptotic effect equivalent to that observed after treatment with low $\mathrm{K}^{+}$.

NP1 overexpression increases the number of cells with active BAX

Because we found that the localization of NP1 in mitochondria evoked by low $\mathrm{K}^{+}$is associated with a reduction in mitochondrial cytochrome $c$ and regulates activation of caspase-3 (Fig. 6), two 
A

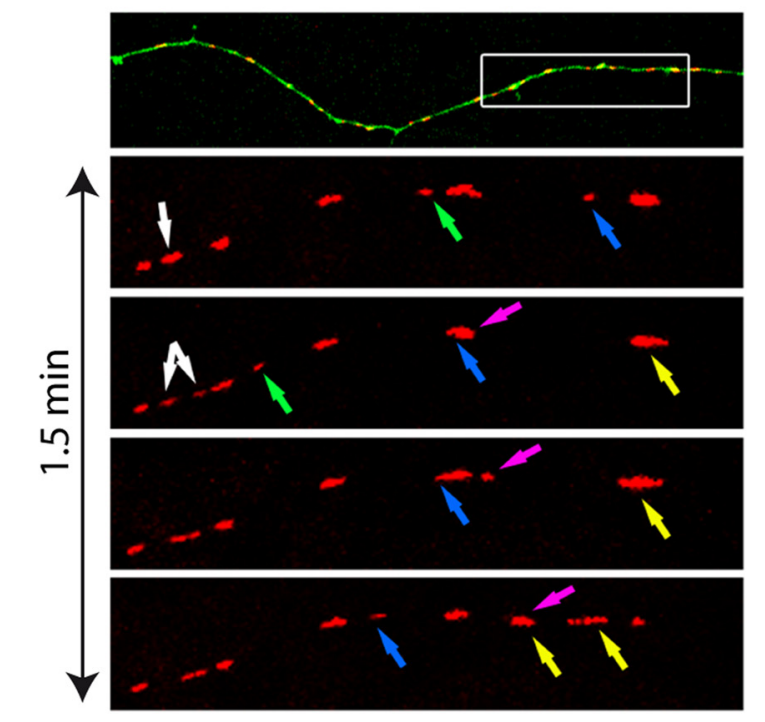

\section{B}

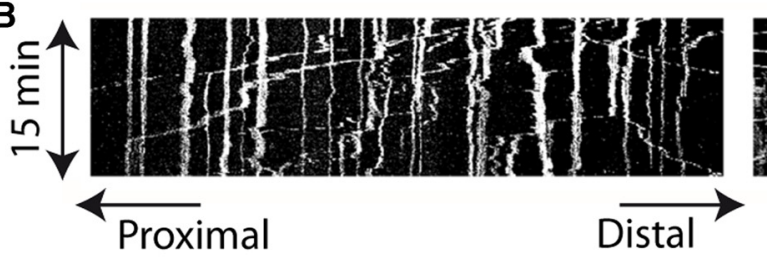

shNP1
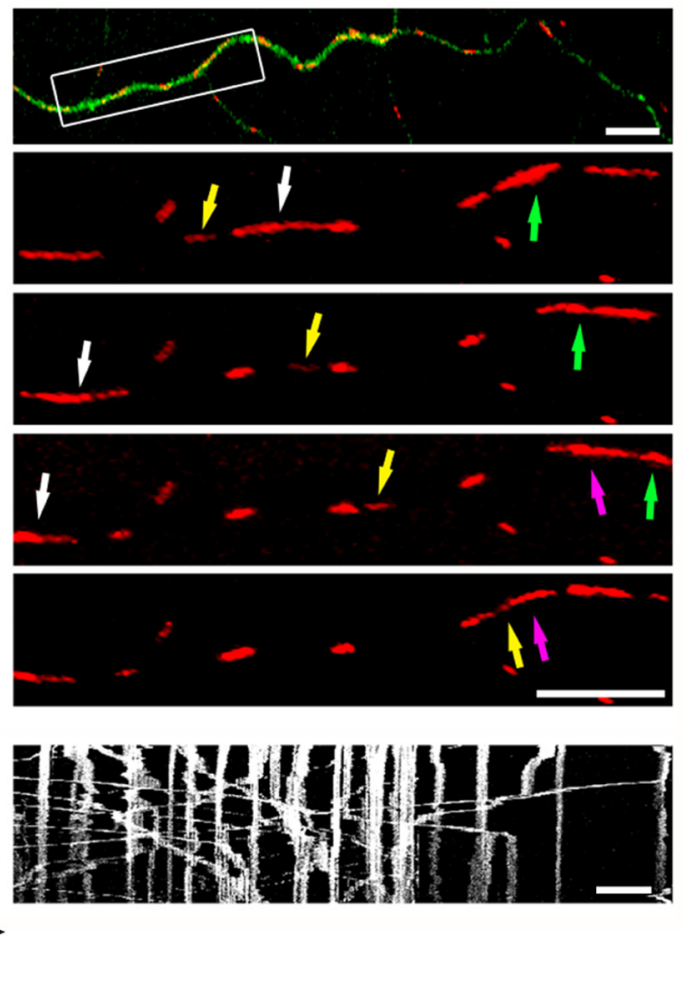

C

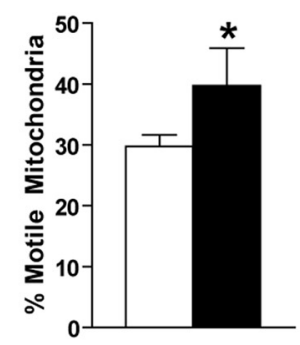

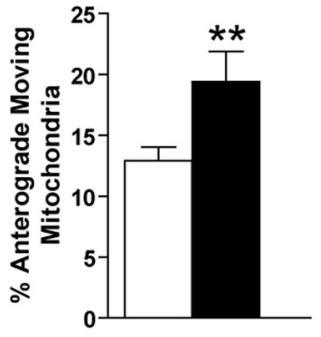
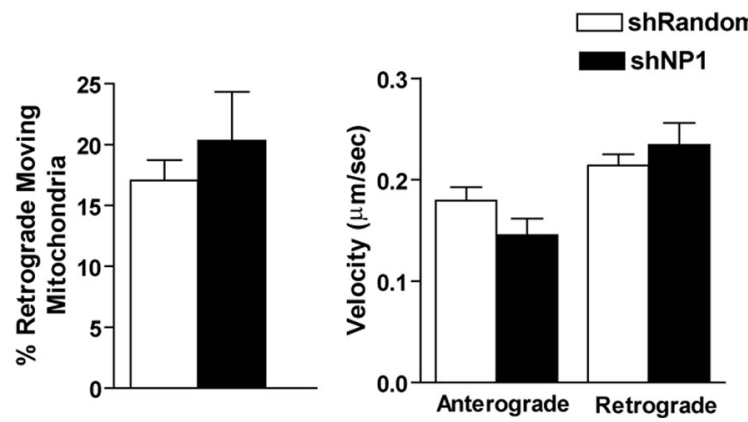

Figure 4. Knockdown of NP1 increases anterograde mitochondrial trafficking. Axonal mitochondrial trafficking was measured in live hippocampal neurons transfected with MtDsRed and shRandom-GFP or shNP1-GFP and recorded $48 \mathrm{~h}$ afterward using a Leica TCS SP2 confocal microscope with a $63 \times$ oil-immersion objective. $A$, The white rectangle in the top panel identifies the section of the imaged axon shown in the image series below from a transfected neuron expressing GFP from shRandom or shNP1 bicistronic vectors. The bottom panels show a series of four confocal images taken every 30 s showing mitochondrial motility in live axons overexpressing MtDsRed and shRandom or shNP1. Colored arrows identify the same mitochondria through the different frames. $B$, Representative kymograph of the full acquisition period (15 min of movie). Kymographs are arranged with the distal axonal end on the right. $C$, Graphs showing quantitative analyses of the percentage age of motile mitochondria and the mitochondrial velocity as measured in the kymographs. Values are mean $\pm \mathrm{SEM} .{ }^{*} p<0.05,{ }^{* *} p<0.01$, significantly different from corresponding shRandom control (independent $t$ test, two tailed, $n=40-50$ axons in each condition). Scale bar, $10 \mu \mathrm{m}$.

processes that require BAX oligomerization, we next studied the influence of NP1 on BAX activation. When active, BAX undergoes a conformational change in the $\mathrm{N}$ terminus that exposes an epitope comprising amino acids 13-19 known as 6A7 (Hsu and Youle, 1998). Using an antibody that recognizes the 6A7 epitope to distinguish active BAX from the other non-active forms, we found that NP1 overexpression significantly increases the number of cells with active BAX $(7 \pm 1 \%$ in cells transduced with NP1 compared with $4 \pm 1 \%$ in controls) (Fig. $7 A, B$ ). In addition, NP1 significantly potentiates the increase in the number of active BAX-expressing cells after $4 \mathrm{~h}$ of treatment with low $\mathrm{K}^{+}(17 \pm$ $2 \%$ in cells transduced with NP1 and treated with low $\mathrm{K}^{+}$compared with $11 \pm 1 \%$ in the respective controls). At the same time, the silencing of NP1 by shRNAi significantly reduced the effect of low $\mathrm{K}^{+}$on active BAX $(7 \pm 1 \%$ in cells transduced with shNP1 and treated with low $\mathrm{K}^{+}$compared with $11 \pm 1 \%$ in the respec- tive controls), indicating that NP1 is required for the increase in active BAX evoked by low $\mathrm{K}^{+}$(Fig. $7 A, B$ ).

\section{NP1 regulates the accumulation of $\mathrm{BAX}$ in mitochondria}

To determine whether NP1 is also involved in the translocation of BAX from cytosol to mitochondria, we studied the effect of NP1 on the accumulation of BAX in mitochondria and on the subcellular redistribution of BAX induced by low $\mathrm{K}^{+}$. In agreement with previous studies (Miller et al., 1997), treatment of CGNs with low $\mathrm{K}^{+}$did not significantly modify the amount of total BAX measured by Western blot when compared with untreated cells (results not shown). Furthermore, consistent with previous results (McGinnis et al., 1999), biochemical experiments measuring the amount of total BAX in subcellular fractions showed that treatment of CGNs with low $\mathrm{K}^{+}$increases the amount of BAX in mitochondria after $2 \mathrm{~h}$ of treatment $(137 \pm 12 \%$ control $)$ (Fig. $7 D)$ but not after $1 \mathrm{~h}$ of treat- 


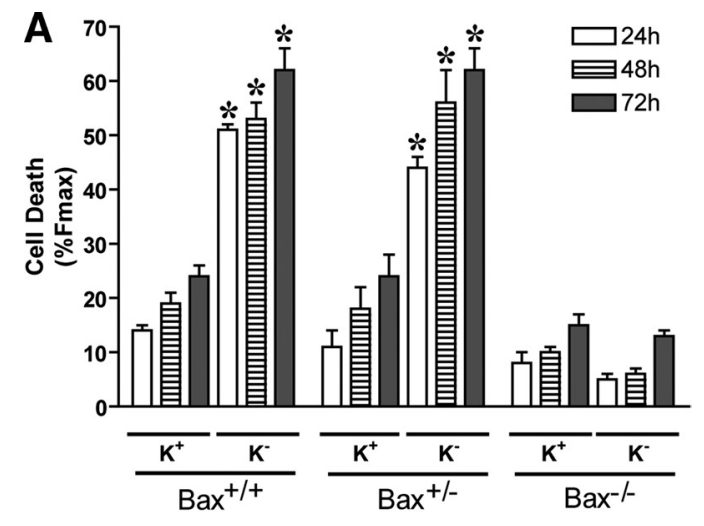

B
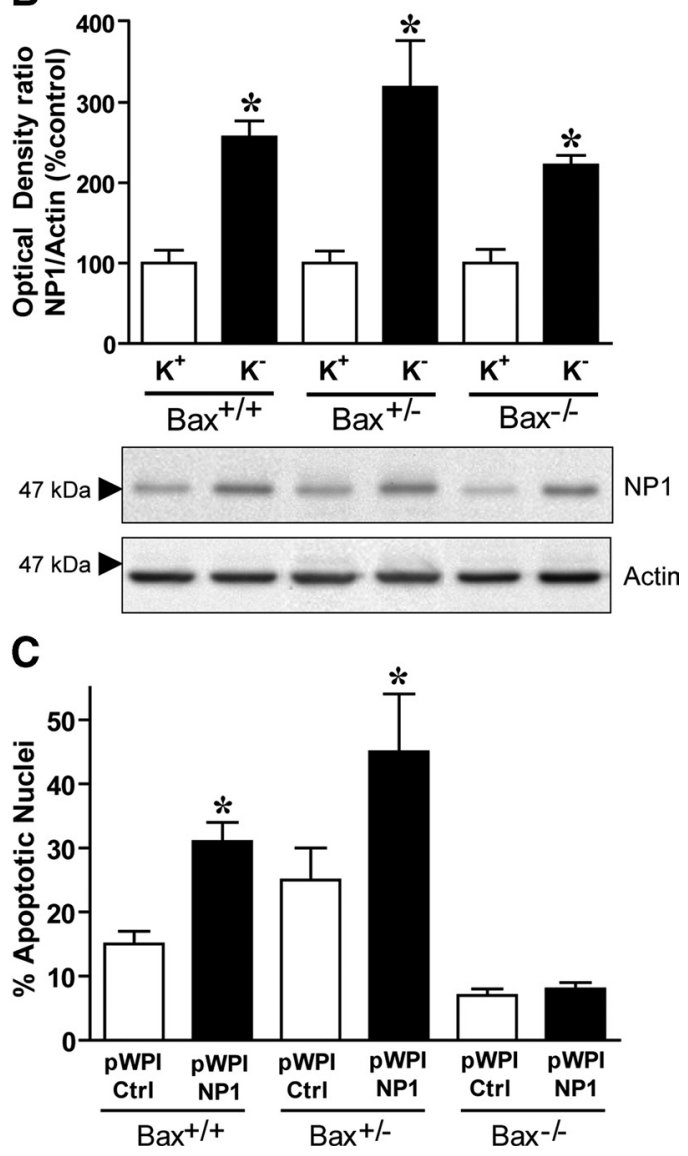

Figure 5. Apoptotic effects of NP1 depend on BAX. Granule cell cultures from wild-type $\left(\mathrm{Bax}^{+/+}\right)$, heterozygote $\left(\mathrm{Bax}^{+/-}\right)$, and homozygote Bax-deficient $\left(\mathrm{Bax}^{-/-}\right)$mice were treated with low $\mathrm{K}^{+}$ $\left(\mathrm{K}^{-}\right)$or with control high $\mathrm{K}^{+}\left(\mathrm{K}^{+}\right)$at DIV8. $A$, Cell death was measured by PI staining. $\mathrm{K}^{+}$deprivation increased cell death in $\mathrm{Bax}^{+/+}$and $\mathrm{Bax}^{+/-}$but not in $\mathrm{Bax}^{-/-}$mice. Values are mean \pm SEM. ${ }^{*} p<0.01$, significantly different from the corresponding high $\mathrm{K}^{+}$(one-way ANOVA with Bonferroni's multiple comparison test, $n=3$ independent experiments). $\boldsymbol{B}$, Quantitative analysis and representative Western blot of NP1 protein expression after $6 \mathrm{~h}$ of low $\mathrm{K}^{+}$treatment in the three Bax genotypes. Reduction of extracellular $\mathrm{K}^{+}$concentration increases NP1 expression in all three genotypes. NP1 was normalized to the levels of actin. The intensity of the bands was determined by densitometric analysis. The rate of NP1 over actin is expressed as percentage of control values. ${ }^{*} p<0.01$, significantly different from corresponding high $\mathrm{K}^{+}$control (one-way ANOVA with Bonferroni's multiple comparison test, $n=5$ independent experiments). C, Granule cells were transduced with the bicistronic lentiviral vector for transgene expression of NP1 (pWPI-NP1) or with the control vector expressing GFP (pWPI-Ctrl). Apoptotic nuclei were assessed with Hoechst 33258 staining. NP1 transduction increases the number of apoptotic nuclei in $\mathrm{Bax}^{+/+}$and $\mathrm{Bax}^{+/-}$mice but not in $\mathrm{Bax}^{-/-}$ animals. Values are mean \pm SEM of $n=6$ independent experiments. The number of animals and cells counted were as follows: $\mathrm{Bax}^{+/+}{ }^{+} n=8$ mice (pWPI-Ctrl, $n=2351$ cells; pWPI-NP1, $n=2971$ cells), $\mathrm{Bax}^{+/-}, n=3$ mice (pWPI-Ctrl, $n=1380$ cells; pWPI-NP1, $n=1606$ cells), Bax ${ }^{-/-}, n=6$ mice (pWPI-Ctrl, $n=4093$ cells; pWPI-NP1, $n=4200$ cells). ${ }^{*} p<0.01$, significantly differentfrom corresponding pWPI-Ctrl (one-way ANOVA with Bonferroni's multiple comparison tests).
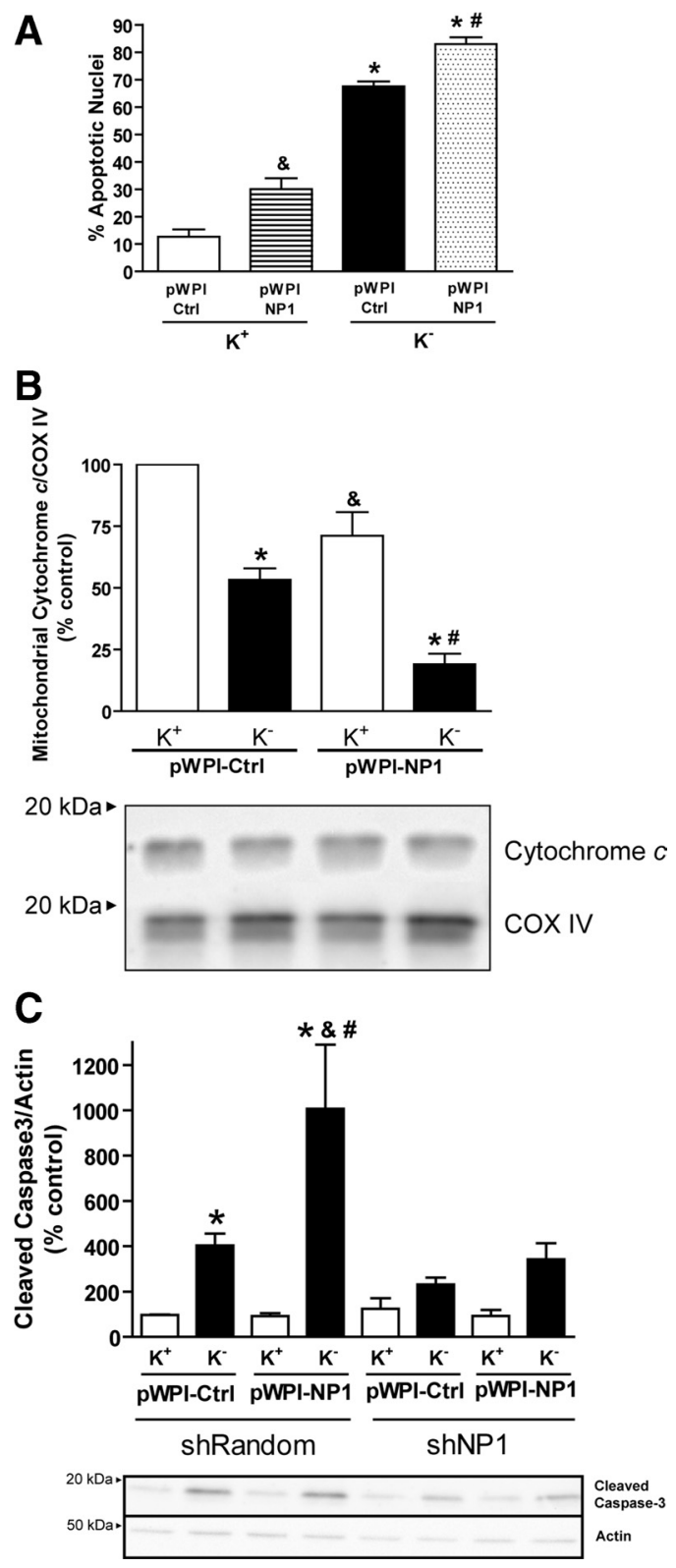

Figure 6. NP1 activates mechanisms of the intrinsic program of apoptosis. Rat CGNs were transduced with control lentiviral vector (pWPI-Ctrl) or with lentiviral vector for transgene expression of NP1 (pWPI-NP1) and treated with low $\mathrm{K}^{+}$. A, Apoptotic nuclei were measured after $24 \mathrm{~h}$ in low $\mathrm{K}^{+}$by Hoechst 33258 staining. Values are mean \pm SEM. ${ }^{\circledR} p<0.05$ and ${ }^{*} p<$ 0.001 , significantly different from $\mathrm{K}^{+} / \mathrm{pWPI}-\mathrm{Ctrl} ;{ }^{*} p<0.05$, significantly different from $\mathrm{K}^{-}$/ pWPI-Ctrl (one-way ANOVA with Bonferroni's multiple comparison tests, $n=3$ ). $\boldsymbol{B}$, Representative Western blot and quantification of mitochondrial cytochrome cover COX IV after low $\mathrm{K}^{+}$ treatment. Lentivirus transduced neurons were treated with low $\mathrm{K}^{+}$for $8 \mathrm{~h}$; mitochondriaenriched fractions were obtained, and cytochrome $\mathrm{c}$ was quantified by densitometric analysis. Transgene expression of NP1 induces cytochrome c release from mitochondria and also potentiates the release evoked by low $\mathrm{K}^{+}$treatment. ${ }^{*} p<0.001$, significantly different from high $\mathrm{K}^{+} / \mathrm{pWPI}-\mathrm{Ctrl} ;{ }^{\&} p<0.01$, significantly different from high $\mathrm{K}^{+} / \mathrm{pWPI-Ctrl;}{ }^{\#} p<0.01$, significantly different from low $\mathrm{K}^{+} / \mathrm{pWPI-Ctrl}$ (one-way ANOVA with Bonferroni's multiple comparison test, $n=8$ ). C, Representative Western blot and densitometric analysis of cleaved caspase-3 over actin after $6-8 \mathrm{~h}$ of low $\mathrm{K}^{+}$treatment at DIV7. Caspase-3 was assayed with rabbit anti-cleaved caspase-3 (Asp 175) that recognizes only the cleaved form. NP1 potentiates the increase of cleaved caspase- 3 evoked by low $\mathrm{K}^{+}$, and this effect is blocked by NP1 knockdown. Values are mean \pm SEM. ${ }^{*} p<0.01$, significantly different from high $\mathrm{K}^{+} \mathrm{pWPI}-\mathrm{Ctrl} /$ shRandom; ${ }^{\&} p<0.001$, significantly different from high $\mathrm{K}^{+} / \mathrm{pWPI}-\mathrm{NP1} /$ shRandom; ${ }^{*} p<$ 0.05 , significantly different from low $\mathrm{K}^{+} / \mathrm{pWPI}-\mathrm{Ctrl} / \mathrm{shRandom}$ (one-way ANOVA with Bonferroni's multiple comparison test, $n=5$ ). 
ment (Fig. 7C). Overexpression of NP1 significantly increased the amount of BAX in mitochondria-enriched subcellular fractions in control high $\mathrm{K}^{+}$-treated cells (128 $\pm 5 \%$ of control values) and potentiated the accumulation of BAX in mitochondria after $1 \mathrm{~h}$ of treatment with low $\mathrm{K}^{+}$ (155 $\pm 5 \%$ of control values) (Fig. 7C). Moreover, knockdown of NP1 blocked the accumulation of BAX in mitochondriaenriched fractions observed after $2 \mathrm{~h}$ of treatment with low $\mathrm{K}^{+}$(Fig. 7D) and prevented the decrease in cytosolic BAX evoked by $8 \mathrm{~h}$ of treatment with low $\mathrm{K}^{+}$(Fig. $7 E$ ). These results indicate that NP1 facilitates and is required for the translocation of $\mathrm{BAX}$ to mitochondria.

NP1 cooperates with BAX in the regulation of mitochondrial length and mass by neuronal activity

Because we found previously that knockdown of NP1 prevents mitochondrial fragmentation evoked by low $\mathrm{K}^{+}$and that NP1 facilitates the translocation of BAX to mitochondria, we asked whether NP1 influences mitochondrial morphology in CGNs from Bax knock-out mice. Confirming the previous results reported in rat CGNs (Fig. 3C), low $\mathrm{K}^{+}$significantly reduced by $\sim 40 \%$ mitochondrial length in wild-type Bax mice $(1.7 \pm 0.1$ and $0.96 \pm 0.03 \mu \mathrm{m}$ in control and low $\mathrm{K}^{+}$. treated wild-type CGNs, respectively) (Fig. 8A). This effect was blocked by cotransduction of the silencing vector for NP1 (Fig. 8A,C). In addition, in untreated CGNs from Bax knock-out mice, mitochondrial length was significantly decreased by $\sim 30 \%$ compared with that in untreated wild-type CGNs $(1.7 \pm 0.1$ and $1.2 \pm 0.04 \mu \mathrm{m}$ in CGNs from $\mathrm{Bax}^{+/+}$ and $\mathrm{Bax}^{-/-}$, respectively) (Fig. $8 A, C$ ). This is consistent with a role for BAX in promoting fusion under basal conditions, which, although shown previously in a number of cell lines and mouse cortical neurons (Karbowski et al., 2006; Cleland et al., 2011), had not been studied previously in CGNs. Furthermore, in CGNs from Bax knock-out mice, reduction of extracellular $\mathrm{K}^{+}$, despite causing a marked increase in NP1 levels (Fig. 5B), did not induce an additional decrease in mitochondrial length (Fig. $8 \mathrm{~A}$ ), implying that NP1 requires $\mathrm{BAX}$ to induce mitochondrial fragmentation under apoptotic conditions. Moreover, knockdown of NP1 in the absence of BAX had no significant effect on mitochondrial length in CGNs maintained in high $\mathrm{K}^{+}$(Fig. $8 A$ ), further confirming that the prevention of mitochondrial fragmentation observed

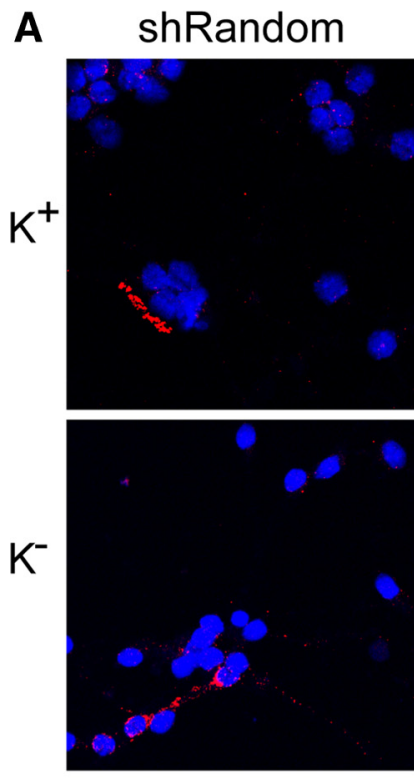

B

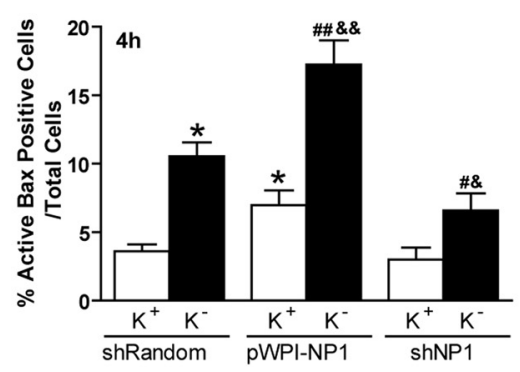

D

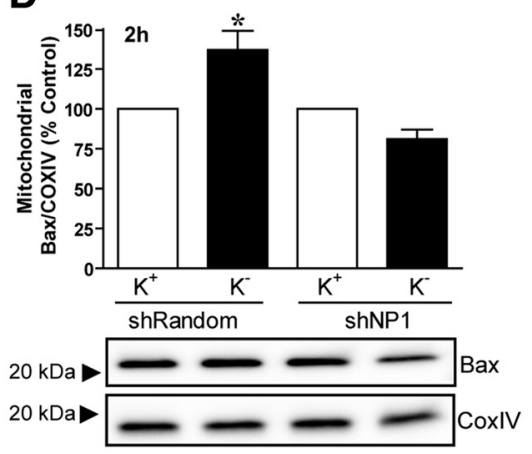

pWPI-NP1
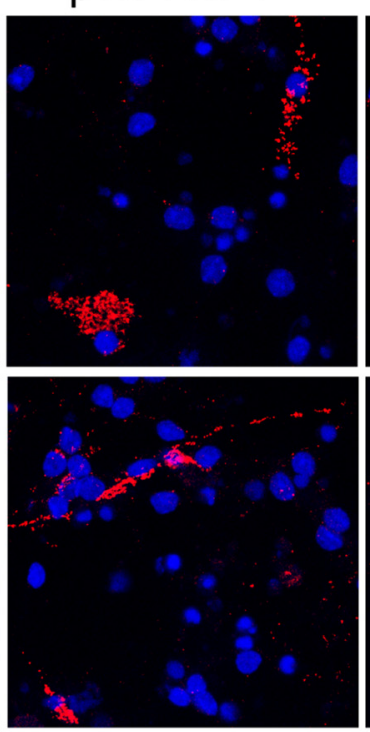

C

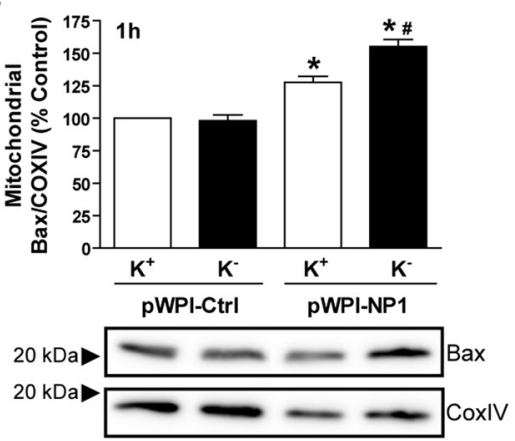

E

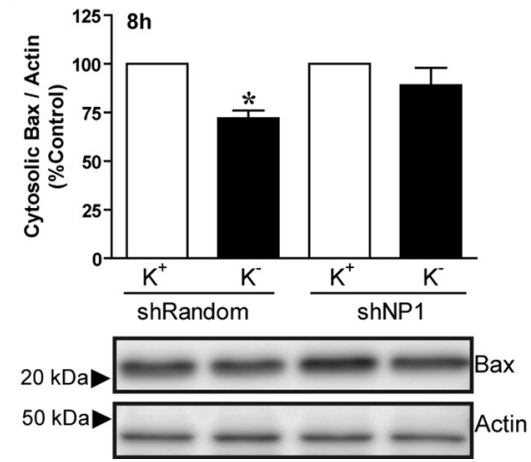

Figure 7. NP1 increases the number of cells with active BAX and contributes to BAX accumulation in mitochondria. CGNs were transduced with control lentiviral vector (pWPI-Ctrl/shRandom) or with the lentiviral vectors for transgene expression of NP1 (pWPI-NP1) or for NP1 knockdown (shNP1) at DIV0. At DIV7, the cells were treated with high or low $\mathrm{K}^{+}$before performing immunocytochemistry or subcellular fractionation. $\boldsymbol{A}$, Representative immunofluorescence confocal images of active BAX and DAPI. Active BAX is shown in red, and cell soma (DAPI) are shown in blue. Scale bar, $20 \mu \mathrm{m}$. $B$, Quantitative analysis of the effect of NP1 and of $4 \mathrm{~h}$ of treatment with low $\mathrm{K}^{+}$on the percentage of cells with active BAX. ${ }^{*} p<0.001$, significantly different from $\mathrm{K}^{+}$/shRandom; ${ }^{\&} p<0.05,{ }^{\& \&} p<0.001$, significantly different from corresponding lentiviral control; ${ }^{\#} p<0.05,{ }^{\# \#} p<0.001$, significantly different from $\mathrm{K}^{-}$/shRandom (two-tailed independent $t$ test, $n=3$ independent cultures,). C, D, Quantitative analyses and representative Western blots of the effects of NP1 overexpression and knockdown on the accumulation of BAX in mitochondria-enriched subcellular fractions. Samples of mitochondria-enriched fractions for detection of BAX by Western blot were obtained $1 \mathrm{~h}(\boldsymbol{C})$ or $2 \mathrm{~h}(\boldsymbol{D})$ after treatment with low $\mathrm{K}^{+}$. The densitometric analysis of BAX was normalized to the corresponding COX IV bands. Values are mean \pm SEM. ${ }^{*} p<0.05$, significantly different from the respective control. ${ }^{*} p<0.05$, significantly different from high $\mathrm{K}^{+} / \mathrm{pWPI-NP1}$ (one-way ANOVA with Bonferroni's multiple comparison test; independent cultures, $n=4$ in $\boldsymbol{C}$ and $n=3$ in $\boldsymbol{D}$ ). $\boldsymbol{E}$, Densitometric analysis and representative Western blot images of BAX and actin from cytosol-enriched fractions obtained after $8 \mathrm{~h}$ of low $\mathrm{K}^{+}$. The densitometric values of BAX bands were normalized to the corresponding actin bands. Values are mean \pm SEM. ${ }^{*} p<0.05$, significantly different from the respective control (one-way ANOVA with Bonferroni's multiple comparison test, $n=6$ independent cultures). 


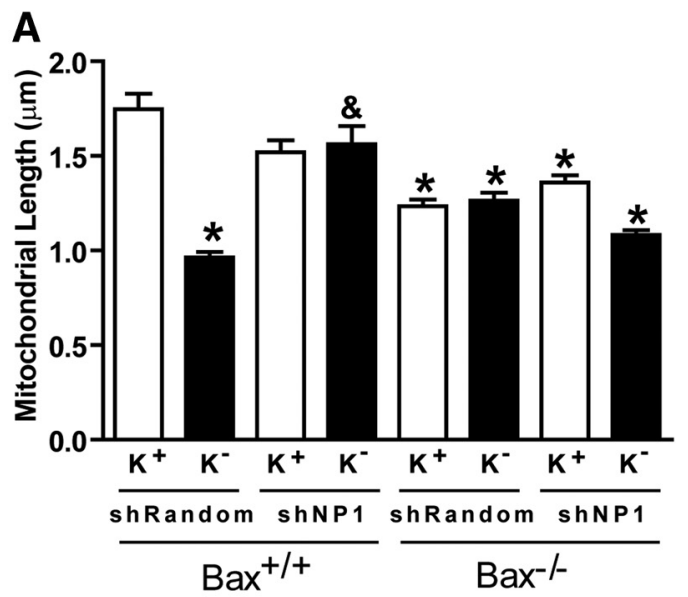

B

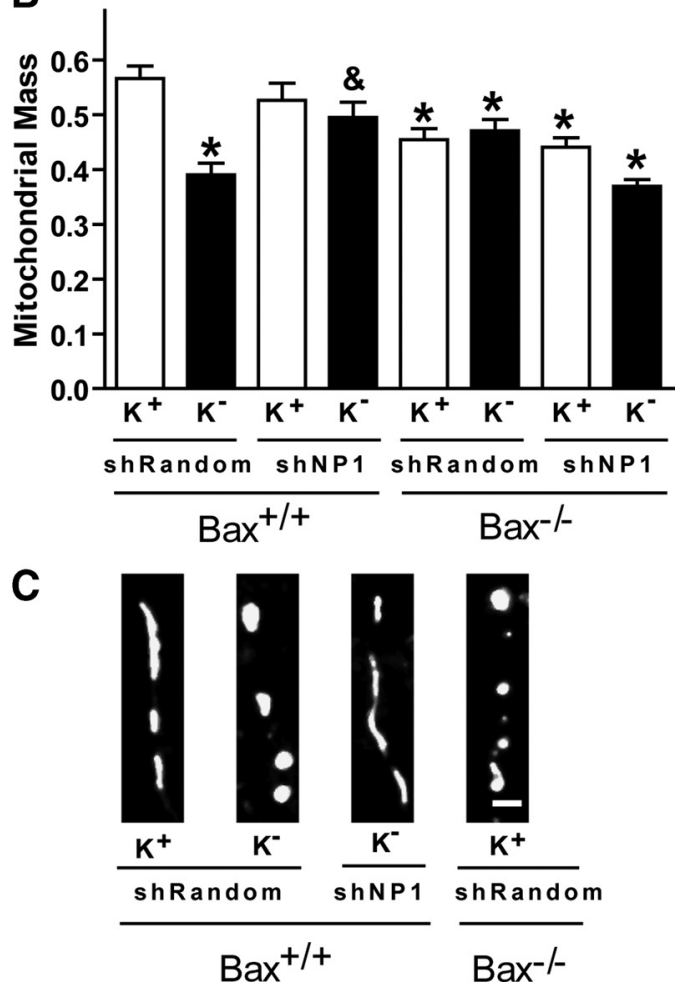

Figure 8. Influence of NP1 and Bax on mitochondrial fragmentation evoked by low $\mathrm{K}^{+}$. CGNs obtained from Bax wild-type and knock-out mice were transduced with control (shRandom) or with the NP1 silencing lentiviral vector (shNP1) on DIV0 and treated with high or low $\mathrm{K}^{+}$for $6 \mathrm{~h}$ on DIV7. A, Influence of NP1 on mitochondrial length in Bax wild types and knockouts in response to low $\mathrm{K}^{+}$. Mitochondria were marked using MtDsRed, and mitochondrial length was measured as described in Materials and Methods. Values are mean \pm SEM. ${ }^{*} p<$ 0.01 , significantly different from high $\mathrm{K}^{+} /$shRandom $\mathrm{Bax}^{+/+}$; ${ }_{\mathrm{i}} \mathrm{p}<0.01$, significantly different from low K ${ }^{+} /$shRandom Bax ${ }^{+/+}$(one-way ANOVA with Bonferroni's multiple comparison tests, $n=$ at least 2 independent cultures, $n=170-450$ mitochondria at each treatment condition). $\boldsymbol{B}$, Mitochondrial mass in neurites from Bax wild types and knock-outs in response to low $\mathrm{K}^{+}$and knockdown of NP1. Mitochondria were marked using MtDsRed, and mitochondrial mass was measured as described in Materials and Methods. Values are mean \pm SEM. ${ }^{*} p<$ 0.01 , significantly different from high $\mathrm{K}^{+} /$shRandom $\mathrm{Bax}^{+/+} ;{ }^{\&} p<0.01$, significantly different from low K ${ }^{+} /$shRandom Bax ${ }^{+/+}$(one-way ANOVA with Bonferroni's multiple comparison tests, $n=$ at least 2 independent cultures, $n=40-80$ mitochondria at each treatment condition). $C$, Representative images of MtDsRed-marked mitochondria in CGNs from Bax wild-type mice transduced with either shRandom or shNP1 and treated with either high $\mathrm{K}^{+}$or low $\mathrm{K}^{+}$ compared with CGNs from Bax knock-outs transduced with shRandom and maintained in high $\mathrm{K}^{+}$. Scale bar, $2 \mu \mathrm{m}$. after NP1 knockdown in low potassium-treated wild-type CGNs is mediated through BAX.

To determine whether the changes in mitochondrial length observed after treatment with low $\mathrm{K}^{+}$and in Bax knock-out mice are associated with changes in individual mitochondrial mass, we next studied the influence of NP1, BAX, and neuronal activity on mitochondrial mass, an index integrating mitochondrial fission, fusion, biogenesis, and degradation (Berman et al., 2009). Consistent with the results observed for mitochondrial length, treatment of wild-type CGNs with low potassium caused a marked reduction of mitochondrial mass, and this effect was prevented by NP1 knockdown (Fig. $8 B$ ). The effect of low potassium on mitochondrial mass was not found in CGNs from Bax knock-out mice, which already showed a significantly reduced mitochondrial mass compared with wild-type controls. These results provide additional evidence indicating that NP1 cooperates with BAX to regulate mitochondrial dynamics during apoptosis.

\section{NP1 interacts with BAX and colocalizes with active BAX in mitochondria}

The interaction of BAX with its putative partner proteins of the BCL2 family can be induced in vitro by nonionic detergents that expose the 6A7 N-terminal epitope of BAX associated with its active conformation (Hsu and Youle, 1997). To investigate a possible interaction between BAX and NP1, we performed immunoprecipitation studies with a $6 \mathrm{~A} 7$ antibody in detergent lysates of both native and recombinant BAX and NP1 proteins. We found that NP1 coimmunoprecipitates with BAX in Triton X-100 lysates from rat brain tissue (Fig. 9A). Similarly, coimmunoprecipitation of BAX and NP1 was detectable in Triton X-100 lysates of CGNs treated with low $\mathrm{K}^{+}$for $2 \mathrm{~h}$ (Fig. 9B). Likewise, in HEK 293T cells expressing recombinant Bax and NP1, BAX coimmunoprecipitated NP1 in Triton X-100 lysates (Fig. 9C). In contrast, we could not detect BAX after immunoprecipitation of NP1 in Triton X-100 lysates from rat brain tissue, CGNs, or HEK 293T cells expressing the recombinant NP1-BAX proteins (results not shown). The inability of NP1 antibody to immunoprecipitate the BAX-NP1 complex could be because its epitope is occupied when NP1 is complexed with BAX or because NP1 only interacts with a fraction of BAX in a subcellular compartment. To test the latter possibility, we performed immunoprecipitation experiments in subcellular fractions treated with CHAPS, a zwitterionic detergent that preserves BAX conformation (Hsu and Youle, 1997). However, we found that, in the presence of CHAPS, the NP1 antibody does not immunoprecipitate NP1 (results not shown). Although these results suggest that NP1 is capable of interacting with $\mathrm{BAX}$, the detergent conditions required for the immunoprecipitation experiments do not allow the study of whether this interaction occurs in a physiological condition. To overcome this limitation, we assessed whether NP1 and BAX are associated in immunocytochemistry studies.

To examine whether BAX and NP1 are associated in mitochondria, we performed studies in CGNs transduced with mitochondrially targeted MtDsRed, in conjunction with double immunostaining with 6A7 and NP1 antibodies to identify active BAX and NP1, respectively (Fig. 9D). After $4 \mathrm{~h}$ of treatment with low $\mathrm{K}^{+}$, confocal images of CGNs reveal that the soma of cells undergoing apoptosis exhibit large clusters of active BAX that colocalize with MtDsRed-labeled mitochondria. Moreover, most of the mitochondria also exhibit NP1 immunofluorescence that at least partially overlaps with the active BAX (Fig. 9D). 
A Brain

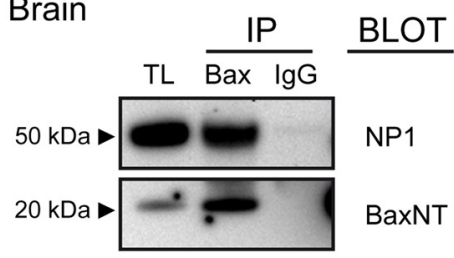

B

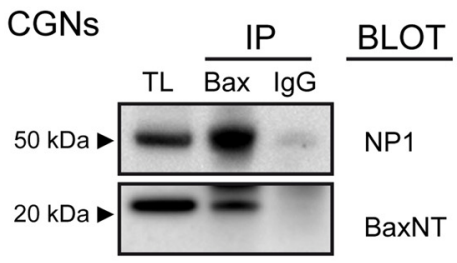

C

293T cells

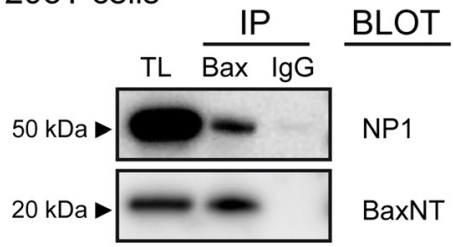

D

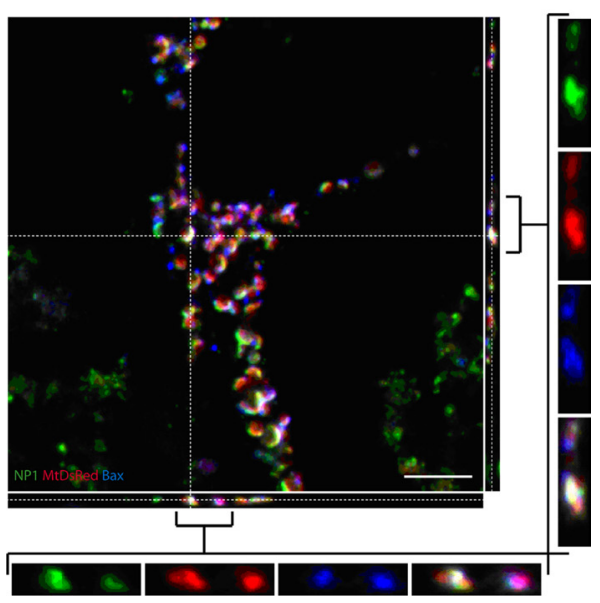

Figure 9. BAX immunoprecipitates NP1 and NP1 colocalizes with active BAX in mitochondria. $\boldsymbol{A - C}$, Representative Western blots of total protein lysate (TL), the eluate of BAX immunoprecipitation (IP; Bax), and the eluate of $\lg G$ immunoprecipitation controls ( $\lg G)$. A, Immunoprecipitation of BAX from total brain lysates. Rat brains were homogenized, and protein lysates prepared as described in Materials and Methods. $\boldsymbol{B}$, Immunoprecipitation of BAX from CGN lysates. CGNs were treated with low $\mathrm{K}^{+}$for $2 \mathrm{~h}$. C, Immunoprecipitation of BAX from HEK 293T cell lysates transfected with recombinant rat Bax and NP1 CDNAs. D, Confocal microscopy studies were performed in mature CGNs in culture after $4 \mathrm{~h}$ of $\mathrm{K}^{+}$deprivation. Orthogonal sectioning shows colocalization between NP1 and active BAX in mitochondria. Colocalization between NP1 and MtDsRed is seen as yellow, between NP1 and BAX as cyan, and between BAX and MtDsRed as magenta, and colocalization between all three proteins is shown in white. Scale bar, $5 \mu \mathrm{m}$.

\section{Discussion}

We have investigated the place of NP1 within the cascade of biochemical events of the mitochondrial apoptosis program. Our findings showing that NP1 facilitates translocation of BAX to mitochondria and regulates mitochondrial dynamics represent new evidence of a non-BCL-2 protein that interacts with BAX and provide novel molecular insight into the mechanism that links the intrinsic program of apoptosis with activity in neurons.
Moreover, the observation that NP1 accumulates in mitochondria after induction of apoptosis in which it regulates mitochondrial fragmentation provides new evidence for an intracellular function of NP1.

In contrast with non-neuronal cell lineages in which there is functional redundancy among multidomain pro-apoptotic proteins of the BCL-2 family, our results confirm previous evidence showing that CGNs require the presence of BAX alone to undergo programmed cell death (Miller et al., 1997). This substantiates the use of the activity-dependent cell death model of CGNs in culture as a reliable model to study the influence of NP1 on the regulation of the BAX-dependent intrinsic program of apoptosis. In this model, our data show that NP1 causes apoptosis by activating mechanisms of the intrinsic program of cell death, such as BAX, cytochrome $c$ release, and caspase-3 (Figs. 6, 7), and that this pro-apoptotic effect of NP1 absolutely requires the presence of BAX. Consequently, these results place NP1 overexpression upstream or in parallel with the activation and/or translocation of BAX to mitochondria.

We found that, during induction of the apoptosis program by low activity, newly synthesized NP1 moves to mitochondria. The finding that treatment of CGNs with low $\mathrm{K}^{+}$increases the amount of NP1 in mitochondria was unexpected because NP2, another member of the same family of pentraxins, has a functional N-terminal secretion signal (Tsui et al., 1996). However, several converging lines of evidence indicate that most of the NP1 induced by low $\mathrm{K}^{+}$is targeted to mitochondria. First, despite the marked increase in the expression of NP1 caused by low $\mathrm{K}^{+}$, we could not detect NP1 released by CGNs in the culture medium. Moreover, biotinylation experiments that label proteins with residues on the extracellular side of the neuronal membrane indicated that the majority of NP1 is intracellular and that only a small fraction of NP1 (5-8\%) is located at the neuronal membrane either before or after treatment of CGNs with low $\mathrm{K}^{+}$. These results are consistent with recent data showing that receptor proteins of the neuronal pentraxin family have an intracellular function (Tseng and Bixby, 2011). Furthermore, biochemical subcellular fractionation studies confirmed that NP1 induced by low $\mathrm{K}^{+}$is preferentially located at the mitochondria-enriched subcellular fraction. Likewise, confocal immunofluorescence analyses of NP1 confirm that, in control CGNs, NP1 is in cytoplasmic puncta not associated with the cell membrane, whereas NP1 increases markedly in mitochondria after treatment with low $\mathrm{K}^{+}$(Fig. 1). In accordance with these findings, substitution of the first 80 amino acids of the NP1 N-terminal side with the corresponding amino acids of NP2 produces a recombinant hybrid protein that, when expressed in HEK $293 \mathrm{~T}$ cells, is found preferentially in the cytosol rather than in the mitochondria-enriched subcellular fraction (results not shown). Overall, these results provide converging evidence to indicate that, after the induction of the intrinsic program of apoptosis by low neuronal activity in CGNs, the majority of NP1 is targeted to the mitochondria.

We next performed a series of studies aimed at identifying the functional effect of the increase of NP1 in mitochondria. We found that NP1 increases the number of cells with active BAX and contributes to the accumulation of BAX to mitochondria (Fig. 7). These results are consistent with the coincidence of time course between the expression of NP1 and the translocation of BAX to mitochondria, which both peak at $4 \mathrm{~h}$ after exposure to low $\mathrm{K}^{+}$in CGNs (McGinnis et al., 1999; DeGregorio-Rocasolano et al., 2001). The maximum level of NP1 expression and BAX translocation to mitochondria lag behind but are near the time of peak 
expression of bim (Putcha et al., 2001) and the point of commitment of CGNs to death, which occurs approximately between 5 and $6 \mathrm{~h}$ after treatment with low $\mathrm{K}^{+}$(Enguita et al., 2005). Because neither NP1 overexpression nor NP1 knockdown modify bim $_{s}$ expression or $\mathrm{BIM}_{\mathrm{EL}}$ protein levels evoked by low $\mathrm{K}^{+}$(results not shown), the present data indicate that the effect of NP1 occurs after the increase in BIM expression and before the accumulation of BAX in mitochondria. The mechanisms of BAX translocation and activation are still under investigation and may vary depending on cell type. The majority of BAX is soluble, resides in the cytoplasm in healthy cells, and, during induction of apoptosis, translocates to the mitochondria (Youle and Strasser, 2008). The finding reported here that knockdown of NP1 prevents the increase in mitochondrial BAX evoked by low $\mathrm{K}^{+}$(Fig. 7) indicates that NP1 acts upstream of BAX and contributes to the activity-dependent process of BAX activation and accumulation in the mitochondria in CGNs. Moreover, the results showing that BAX coimmunoprecipitates NP1, both the endogenous protein from brain tissue and cultured CGNs, as well as recombinant BAX (Fig. 9), suggest that NP1 forms a protein complex with BAX that facilitates its activation and accumulation in mitochondria. In support of this interpretation, our data from confocal immunofluorescence analyses show that NP1 colocalizes with clusters of active BAX in mitochondria (Fig. 9).

In addition to its role in apoptosis, BAX regulates mitochondrial fusion-fission dynamics through a direct interaction with proteins involved in mitochondrial morphogenesis (Sheridan et al., 2008; Cleland et al., 2011; Hoppins et al., 2011). Based on our finding that NP1 interacts with BAX, we investigated the influence of NP1 on mitochondrial length and we found that NP1 also regulates activity-dependent mitochondrial dynamics. Exposure of CGNs to low $\mathrm{K}^{+}$increased the number of NP1 puncta at putative mitochondrial scission sites and significantly reduced by $30-40 \%$ neuritic mitochondrial length (Fig. 2). Knockdown of NP1 blocked this fragmentation (Figs. 3, 8), showing that NP1 is required for the mitochondrial fragmentation evoked by low activity. Based on these results, we next sought to determine whether the effect of NP1 on mitochondrial dynamics is BAX dependent. Thus, we studied the influence of NP1 and BAX on mitochondrial length in cultured CGNs from mice with different gene dosages of Bax. In agreement with the results reported in Figures $2 A$ and $3 C$ that were obtained in cultured rat CGNs, treatment of wild-type mouse CGNs with low $\mathrm{K}^{+}$caused a marked reduction in mitochondrial length and mass. These effects were completely blocked by the knockdown of NP1 (Fig. 8), confirming that NP1 is required for mitochondrial fragmentation caused by low neuronal activity. Moreover, consistent with previous studies (Karbowski et al., 2006), mitochondria in CGNs from Bax knock-out mice were fragmented, and, in these cells, induction of apoptosis by low $\mathrm{K}^{+}$did not lead to an additional reduction in mitochondrial length (Fig. 8). In addition, knockdown of NP1 did not prevent the mitochondrial fragmentation observed in Bax knock-out mice. These results indicate that NP1 contributes to mitochondrial fragmentation during apoptosis by facilitating the activation of BAX and neutralizing in turn the positive effect that soluble BAX has on mitochondrial fusion in healthy cells (Hoppins et al., 2011). This interpretation is consistent with the mechanistic model that proposes a mutually exclusive relationship between the role of BAX in the positive regulation of mitochondrial fusion and its role in apoptosis (Sheridan et al., 2008; Cleland et al., 2011; Hoppins et al., 2011). Overall, the present results indicate that NP1 is required for mitochondrial fragmentation during apoptosis and suggest that activity-dependent regulation of NP1 expression is part of a mechanism that transforms the physiological action of proteins that regulate mitochondrial dynamics in healthy cells such as BAX into a pathological pro-apoptotic function.

In neurons, mitochondrial length may also influence subcellular mitochondrial distribution; the transport of mitochondria to synapses in which there is a high energy demand is essential for neuronal viability. We found that, in addition to its effect on mitochondrial morphology, NP1 reduces anterograde, but not retrograde, movement of mitochondria without altering their velocity (Fig. 4). Other pro-apoptotic proteins associated with neurodegenerative diseases, such as mutant forms of Huntingtin and superoxide dismutase as well as $\mathrm{A} \beta$, have been reported to induce mitochondrial fragmentation and impair mitochondrial trafficking (for review, see Rintoul and Reynolds, 2010). Although the relationship between mitochondrial dynamics and transport is still not well understood, the observation that NP1 interacts with BAX, which interacts in turn with Mitofusin 2 (Hoppins et al., 2011), a protein that is necessary for mitochondrial transport through Miro 1, a GTPase localized in mitochondria (Misko et al., 2010), suggests that NP1 may interfere with anterograde transport by preventing the association of BAX with the Miro/Milton protein complex. This hypothesis is currently under investigation.

In summary, we report that the intrinsic program of neuronal apoptosis evoked by low neuronal activity depends on the overexpression of NP1 that is targeted to mitochondria. The increased expression of NP1 during apoptosis occurs upstream of BAX and facilitates the activation and accumulation of BAX in mitochondria. Moreover, NP1 regulates mitochondrial trafficking and is necessary for mitochondrial fragmentation evoked by low neuronal activity. These data provide evidence for a novel function for NP1 in the regulation of mitochondrial dynamics and trafficking and indicate that NP1 expression and targeting to the mitochondria during apoptosis is part of the mechanism by which low neuronal activity regulates the mitochondrial program of apoptosis. Moreover, because NP1 is markedly increased in brain tissue from patients with late-onset Alzheimer's disease (Abad et al., 2006), the present results may be of importance in understanding the mechanisms of neuronal dysfunction in chronic neurodegenerative disorders that have been associated with alterations in mitochondrial function.

\section{References}

Abad MA, Enguita M, DeGregorio-Rocasolano N, Ferrer I, Trullas R (2006) Neuronal pentraxin 1 contributes to the neuronal damage evoked by amyloid-beta and is overexpressed in dystrophic neurites in Alzheimer's brain. J Neurosci 26:12735-12747.

Autret A, Martin SJ (2009) Emerging role for members of the Bcl-2 family in mitochondrial morphogenesis. Mol Cell 36:355-363.

Berman SB, Chen YB, Qi B, McCaffery JM, Rucker EB 3rd, Goebbels S, Nave KA, Arnold BA, Jonas EA, Pineda FJ, Hardwick JM (2009) Bcl-x L increases mitochondrial fission, fusion, and biomass in neurons. J Cell Biol 184:707-719.

Cleland MM, Norris KL, Karbowski M, Wang C, Suen DF, Jiao S, George NM, Luo X, Li Z, Youle RJ (2011) Bcl-2 family interaction with the mitochondrial morphogenesis machinery. Cell Death Differ 18:235-247.

DeGregorio-Rocasolano N, Gasull T, Trullas R (2001) Overexpression of neuronal pentraxin 1 is involved in neuronal death evoked by low $\mathrm{K}^{+}$in cerebellar granule cells. J Biol Chem 276:796-803.

D’Mello SR, Galli C, Ciotti T, Calissano P (1993) Induction of apoptosis in cerebellar granule neurons by low potassium: inhibition of death by insulin-like growth factor-I and cAMP. Proc Natl Acad Sci U S A 90:10989-10993.

Enguita M, DeGregorio-Rocasolano N, Abad A, Trullas R (2005) Glycogen synthase kinase 3 activity mediates neuronal pentraxin 1 expression and 
cell death induced by potassium deprivation in cerebellar granule cells. Mol Pharmacol 67:1237-1246.

Galluzzi L, Blomgren K, Kroemer G (2009) Mitochondrial membrane permeabilization in neuronal injury. Nat Rev Neurosci 10:481-494.

Hoppins S, Edlich F, Cleland MM, Banerjee S, McCaffery JM, Youle RJ, Nunnari J (2011) The soluble form of Bax regulates mitochondrial fusion via MFN2 homotypic complexes. Mol Cell 41:150-160.

Hsu YT, Youle RJ (1997) Nonionic detergents induce dimerization among members of the Bcl-2 family. J Biol Chem 272:13829-13834.

Hsu YT, Youle RJ (1998) Bax in murine thymus is a soluble monomeric protein that displays differential detergent-induced conformations. J Biol Chem 273:10777-10783.

Karbowski M, Norris KL, Cleland MM, Jeong SY, Youle RJ (2006) Role of Bax and Bak in mitochondrial morphogenesis. Nature 443:658-662.

Loucks FA, Schroeder EK, Zommer AE, Hilger S, Kelsey NA, Bouchard RJ, Blackstone C, Brewster JL, Linseman DA (2009) Caspases indirectly regulate cleavage of the mitochondrial fusion GTPase OPA1 in neurons undergoing apoptosis. Brain Res 1250:63-74.

Macaskill AF, Rinholm JE, Twelvetrees AE, Arancibia-Carcamo IL, Muir J, Fransson A, Aspenstrom P, Attwell D, Kittler JT (2009) Mirol is a calcium sensor for glutamate receptor-dependent localization of mitochondria at synapses. Neuron 61:541-555.

McGinnis KM, Gnegy ME, Wang KK (1999) Endogenous bax translocation in SH-SY5Y human neuroblastoma cells and cerebellar granule neurons undergoing apoptosis. J Neurochem 72:1899-1906.

Miller KE, Sheetz MP (2004) Axonal mitochondrial transport and potential are correlated. J Cell Sci 117:2791-2804.

Miller TM, Moulder KL, Knudson CM, Creedon DJ, Deshmukh M, Korsmeyer SJ, Johnson EM Jr (1997) Bax deletion further orders the cell death pathway in cerebellar granule cells and suggests a caspaseindependent pathway to cell death. J Cell Biol 139:205-217.

Misko A, Jiang S, Wegorzewska I, Milbrandt J, Baloh RH (2010) Mitofusin 2 is necessary for transport of axonal mitochondria and interacts with the Miro/Milton complex. J Neurosci 30:4232-4240.

Putcha GV, Deshmukh M, Johnson EM Jr (1999) BAX translocation is a critical event in neuronal apoptosis: regulation by neuroprotectants, BCL-2, and caspases. J Neurosci 19:7476-7485.

Putcha GV, Moulder KL, Golden JP, Bouillet P, Adams JA, Strasser A, Johnson EM (2001) Induction of BIM, a proapoptotic BH3-only BCL-2 family member, is critical for neuronal apoptosis. Neuron 29:615-628.

Rintoul GL, Reynolds IJ (2010) Mitochondrial trafficking and morphology in neuronal injury. Biochim Biophys Acta 1802:143-150.

Schlimgen AK, Helms JA, Vogel H, Perin MS (1995) Neuronal pentraxin, a secreted protein with homology to acute phase proteins of the immune system. Neuron 14:519-526.

Sheridan C, Delivani P, Cullen SP, Martin SJ (2008) Bax- or Bak-induced mitochondrial fission can be uncoupled from cytochrome $\mathrm{C}$ release. $\mathrm{Mol}$ Cell 31:570-585.

Suen DF, Norris KL, Youle RJ (2008) Mitochondrial dynamics and apoptosis. Genes Dev 22:1577-1590.

Tseng LA, Bixby JL (2011) Interaction of an intracellular pentraxin with a BTB-Kelch protein is associated with ubiquitylation, aggregation and neuronal apoptosis. Mol Cell Neurosci 47:254-264.

Tsui CC, Copeland NG, Gilbert DJ, Jenkins NA, Barnes C, Worley PF (1996) Narp, a novel member of the pentraxin family, promotes neurite outgrowth and is dynamically regulated by neuronal activity. J Neurosci 16:2463-2478.

Youle RJ, Strasser A (2008) The BCL-2 protein family: opposing activities that mediate cell death. Nat Rev Mol Cell Biol 9:47-59. 